\title{
ROOTED EDGES OF A MINIMAL DIRECTED SPANNING TREE ON RANDOM POINTS
}

\author{
Z. D. BAI, ${ }^{*}$ Northeast Normal University and National University of Singapore \\ SUNGCHUL LEE, ${ }^{* *}$ Yonsei University \\ MATHEW D. PENROSE, ${ }^{* * *}$ University of Bath
}

\begin{abstract}
For $n$ independent, identically distributed uniform points in $[0,1]^{d}, d \geq 2$, let $L_{n}$ be the total distance from the origin to all the minimal points under the coordinatewise partial order (this is also the total length of the rooted edges of a minimal directed spanning tree on the given random points). For $d \geq 3$, we establish the asymptotics of the mean and the variance of $L_{n}$, and show that $L_{n}$ satisfies a central limit theorem, unlike in the case $d=2$.
\end{abstract}

Keywords: Minimal spanning tree; multivariate extreme; central limit theorem

2000 Mathematics Subject Classification: Primary 60D05; 60G70

Secondary 05C80; 60F05

\section{Introduction and statement of results}

For $d \geq 2$, let ' $\prec$ ' denote the coordinatewise partial order on $\mathbb{R}^{d}: \boldsymbol{x} \prec \boldsymbol{y}$ if and only if all coordinates of $\boldsymbol{y}-\boldsymbol{x}$ are nonnegative and $\boldsymbol{x} \neq \boldsymbol{y}$. For $S \subset \mathbb{R}^{d}$ and $\boldsymbol{x} \in S$, we say that $\boldsymbol{x}$ is a minimal element of $S$ if no $\boldsymbol{y} \in S$ satisfies $\boldsymbol{y} \prec \boldsymbol{x}$, and that $\boldsymbol{x}$ is a maximal element of $S$ if no $\boldsymbol{y} \in S$ satisfies $\boldsymbol{x} \prec \boldsymbol{y}$. Let $\mathcal{M}(S)$ denote the set of minimal elements of $S$. In this paper, our major interest is in $\mathcal{M}(S)$, where $S$ is a random set $\mathcal{X}_{n}$ consisting of $n$ independent, identically distributed uniform points in $[0,1]^{d}, d \geq 3$. More precisely, we study the asymptotics of the random variables $L_{n}$ given by

$$
L_{n}:=\sum_{\boldsymbol{x} \in \mathcal{M}\left(\mathcal{X}_{n}\right)}|\boldsymbol{x}|,
$$

where $|\cdot|$ denotes the Euclidean norm.

The quantity $L_{n}$ arises in the context of a certain spanning tree problem, which we now describe. Suppose that $S$ is a finite subset of $[0,1]^{d}$ and let $\mathbf{0}$ denote the origin of $\mathbb{R}^{d}$. Then $\mathbf{0}$ is the only minimal element of $S \cup\{\mathbf{0}\}$. A directed spanning tree on $S \cup\{\mathbf{0}\}$ is a directed graph $G$ with vertex set $S \cup\{\boldsymbol{0}\}$, such that (i) all directed edges are of the form $(\boldsymbol{x}, \boldsymbol{y})$ with $\boldsymbol{y} \prec \boldsymbol{x}$, and (ii) for every $\boldsymbol{x} \in S$ there is a unique directed path in $G$ from $\boldsymbol{x}$ to $\mathbf{0}$. The length of $G$, denoted $L(G)$, is the sum of the Euclidean lengths of its edges. A minimal directed spanning tree on $S \cup\{\mathbf{0}\}$ is a directed spanning tree $G$ with the property that $L(G) \leq L\left(G^{\prime}\right)$ for every

Received 28 September 2004; revision received 6 July 2005.

* Postal address: School of Mathematics and Statistics, Northeast Normal University, Changchun, 130024, P. R. China.

** Postal address: Department of Mathematics, Yonsei University, Seoul 120-749, Korea.

Email address: sungchul@yonsei.ac.kr

*** Postal address: Department of Mathematical Sciences, University of Bath, Bath BA2 7AY, UK. 
other directed spanning tree $G^{\prime}$ on $S \cup\{\mathbf{0}\}$. It can be shown that the minimal directed spanning tree on $\mathcal{X}_{n} \cup\{\boldsymbol{0}\}$ is almost surely unique.

The study of minimal directed spanning trees on random points was initiated by Bhatt and Roy [6], motivated by applications to communications and drainage networks. The construction of the minimal directed spanning tree resembles that of other graphs in which edges are drawn between nearby points in Euclidean space, such as the 'ordinary' minimal spanning tree, the nearest-neighbor graph, and the geometric graph. The probability theory of graphs of this type on random points is well developed; see, for example, [12], [13], [15], [16], [17], and [18]. However, the minimal directed spanning tree has some distinctive features, notably that there is no uniform bound on vertex degrees, and the presence of significant boundary effects. In view of these features, it is a reasonable first step to consider the rooted edges of the minimal directed spanning tree, i.e. those edges that are incident at the origin.

For $\boldsymbol{x} \in \mathcal{M}(S)$, the edge $(\boldsymbol{x}, \mathbf{0})$ is in any directed spanning tree on $S \cup\{\boldsymbol{0}\}$. Conversely, if $\boldsymbol{x} \in S$ with $(\boldsymbol{x}, \mathbf{0})$ an edge of a minimal directed spanning tree $G$ on $S \cup\{\boldsymbol{0}\}$, then $\boldsymbol{x}$ must be in $\mathcal{M}(S)$ (since otherwise we could find a $\boldsymbol{y} \in \mathcal{M}(S)$ with $\boldsymbol{y} \prec \boldsymbol{x}$ and improve on the length of $G$ by replacing the edge $(\boldsymbol{x}, \mathbf{0})$ by the edge $(\boldsymbol{x}, \boldsymbol{y}))$. Consequently, the set of rooted edges of a minimal directed spanning tree on $S \cup\{\mathbf{0}\}$ is precisely the set of edges $(\boldsymbol{x}, \mathbf{0}), \boldsymbol{x} \in \mathcal{M}(S)$.

The number of rooted edges is hence precisely the number of minimal elements of $S$, which we denote $|\mathcal{M}(S)|$. This quantity is of interest in multivariate extreme-value theory, and the probability theory of $\left|\mathcal{M}\left(\mathcal{X}_{n}\right)\right|$ has received a degree of recent attention (see [1], [3], and references therein). In particular, Bai et al. [2] recently established that $\left|\mathcal{M}\left(\mathcal{X}_{n}\right)\right|$ satisfies a central limit theorem for $d \geq 2$. (Actually, they considered the number of maxima in $\mathcal{X}_{n}$, which obviously has the same distribution as the number of minima.)

In the present work, we are instead concerned with the quantity $L_{n}$ defined in (1), which is the total length of the rooted edges of the minimal directed spanning tree on $\mathcal{X}_{n}$. In the case $d=2$, Bhatt and Roy [6] showed that the distribution of $L_{n}$ converges weakly to a certain limiting distribution, with corresponding convergence of all moments; subsequently, Penrose and Wade [14] identified the limiting distribution as a type of Dickman distribution. It is clear that this limiting distribution is nonnormal since it is supported on the half-line $[0, \infty)$ (no rescaling or centering of $L_{n}$ is required in Bhatt and Roy's result).

Thus, for $d=2$ there is a distinction between the limiting distribution of $L_{n}$, which is not normal, and that of a renormalized version of $\left|\mathcal{M}_{n}\right|$, which is normal. This distinction is essentially due to the effect of long edges. It is natural to ask whether this distinction persists in higher dimensions, and in this paper we answer this question in the negative by showing that, for $d \geq 3$, the limiting distribution of $L_{n}$ (suitably scaled and centered) is indeed normal, using a method related to that of [2]. Moreover, we give precise asymptotic expressions for the mean and variance of $L_{n}$.

As a final introductory remark, we note that there is a resemblance between the study of minimal elements of a random sample, as in the present paper, and the study of convex hulls of random samples. In the latter subject, quite a lot is known [7], [8], [10] for $d=2$, but much less is known in higher dimensions, as far as the authors are aware.

In this paper, we write $A_{n} \asymp B_{n}$ to express the fact that $A_{n}=B_{n}\left(1+O\left((\log n)^{-1}\right)\right)$.

Here are the precise asymptotic expressions for the mean and variance of $L_{n}$.

Theorem 1. For $d \geq 3$, as $n \rightarrow \infty$,

$$
\mathrm{E}\left(L_{n}\right) \asymp \frac{d}{(d-2) !}(\log n)^{d-2} .
$$


Theorem 2. For $d \geq 3$, as $n \rightarrow \infty$,

$$
\operatorname{var}\left(L_{n}\right) \asymp\left(\frac{1}{2} \frac{d}{(d-2) !}+2 \sum_{k=1}^{d-1}\left(\begin{array}{l}
d \\
k
\end{array}\right) k h_{k}-\gamma_{d}\right)(\log n)^{d-2},
$$

where $h_{k}, 1 \leq k \leq d-1$, and $\gamma_{d}\left(\gamma_{d}<d /(2(d-2) !)\right)$ are strictly positive finite constants: for $k=1$,

$$
\begin{array}{rl}
h_{1}=\int_{0}^{1} & \mathrm{~d} w_{1} \int_{0}^{1} \mathrm{~d} w_{2} w_{1}\left(\left(w_{1}+w_{2}-w_{1} w_{2}\right)^{-2}-\left(w_{1}+w_{2}\right)^{-2}\right) \\
& \times \frac{1}{2} \frac{1}{(d-2) !} \frac{\left(-\log w_{2}\right)^{d-2}}{(d-2) !}
\end{array}
$$

for $2 \leq k \leq d-1$,

$$
\begin{gathered}
h_{k}=\int_{0}^{1} \mathrm{~d} u_{1} \int_{0}^{u_{1}} \mathrm{~d} w_{1} \int_{0}^{1} \mathrm{~d} w_{2}\left(\left(w_{1}+w_{2}-w_{1} w_{2}\right)^{-2}-\left(w_{1}+w_{2}\right)^{-2}\right) \\
\times \frac{1}{2} \frac{1}{(d-2) !} \frac{\left(-\log w_{1}+\log u_{1}\right)^{k-2}}{(k-2) !} \frac{\left(-\log w_{2}\right)^{d-k-1}}{(d-k-1) !},
\end{gathered}
$$

and

$$
\begin{aligned}
\gamma_{d} & =\frac{d}{((d-2) !)^{2}}\left(\int_{0}^{1} \mathrm{~d} v_{1} \int_{0}^{1} \mathrm{~d} s \frac{1}{\left(1+v_{1} s\right)^{2}}\left(\log \frac{1}{s}\right)^{d-2} v_{1}\right) \\
& <\frac{d}{((d-2) !)^{2}}\left(\int_{0}^{1} \mathrm{~d} v_{1} \int_{0}^{1} \mathrm{~d} s\left(\log \frac{1}{s}\right)^{d-2} v_{1}\right) \\
& =\frac{1}{2} \frac{d}{(d-2) !} .
\end{aligned}
$$

Our final result, Theorem 3, is a central limit theorem for $L_{n}$. To state it, we introduce the following notation: we write $Y_{n} \in \operatorname{CLT}\left(r_{n}\right)$ if

$$
\sup _{x}\left|\mathrm{P}\left(\frac{Y_{n}-\mathrm{E}\left(Y_{n}\right)}{\left(\operatorname{var}\left(Y_{n}\right)\right)^{1 / 2}} \leq x\right)-\Phi(x)\right|=O\left(r_{n}\right) \quad \text { and } \quad r_{n} \rightarrow 0,
$$

where $\Phi(x)$ is the cumulative distribution function for the standard normal distribution.

Theorem 3. For $d \geq 3$, as $n \rightarrow \infty$,

$$
\frac{L_{n}-\mathrm{E}\left(L_{n}\right)}{\left(\operatorname{var}\left(L_{n}\right)\right)^{1 / 2}} \rightarrow N(0,1)
$$

in distribution. In fact, we have

$$
L_{n} \in \operatorname{CLT}\left((\log n)^{-(d-2) / 4}(\log \log n)^{(d+1) / 2}\right) .
$$

In Sections 2 and 3 we prove Theorems 1 and 2, respectively. We write the mean and the variance of $L_{n}$ exactly as integrals and, using two elementary but useful inequalities, (9) and (10), approximate the exact integrals by more tractable ones. By evaluating the tractable integrals we obtain the asymptotic expressions (2) and (3). 
In Section 4 we prove Theorem 3. With the help of a certain transformation, we approximate $L_{n}$ by a space-truncated random variable conditioned on a highly probable event. We then approximate this conditioned, space-truncated random variable by a random variable $L_{n}^{\prime \prime}$ generated by a Poisson point process. By decomposing $L_{n}^{\prime \prime}$ as a sum of locally dependent random variables, we can apply Stein's method to $L_{n}^{\prime \prime}$ to obtain the central limit theorem for $L_{n}^{\prime \prime}$. Since our approximation errors turn out to be small, we can extract the central limit theorem for $L_{n}$ (Theorem 3) from the central limit theorem for $L_{n}^{\prime \prime}$. Throughout the paper many strictly positive, finite constants whose specific values are irrelevant will appear; we generically denote them by $C$.

Full details of our calculations can be found at http://math.yonsei.ac.fr/sungchul.

\section{Expectation}

Let $\mathcal{X}_{n}$ be the collection $\left\{\boldsymbol{x}_{1}, \ldots, \boldsymbol{x}_{n}\right\}$ of independent, identically distributed uniform points on $[0,1]^{d}, d \geq 3$. Given $\mathcal{X}_{n}$, denote the event that $\boldsymbol{x}_{i}$ is minimal in $\mathcal{X}_{n}$ by $G_{i}$. Then we can rewrite $L_{n}$ as

$$
L_{n}=\sum_{i=1}^{n}\left|\boldsymbol{x}_{i}\right| 1_{G_{i}},
$$

where $1_{A}$ is the indicator function for the set $A$.

In this section we prove Theorem 1. Using (7) we write $\mathrm{E}\left(L_{n}\right)$ as an explicit integral, (8). Using two elementary but useful inequalities, (9) and (10), we approximate the explicit integral as a more tractable integral. By evaluating this integral we recover Theorem 1.

By (7) and the exchangeability of the $\boldsymbol{x}_{i}$, we have

$$
\mathrm{E}\left(L_{n}\right)=n \mathrm{E}\left(\left|x_{1}\right| 1_{G_{1}}\right) .
$$

For $\boldsymbol{x}_{1}:=\left(x_{1}, \ldots, x_{d}\right)$ to be a minimal point (i.e. $\left.1_{G_{1}}=1\right)$, all the other points $\boldsymbol{x}_{j}, 2 \leq j \leq n$, should avoid the region 'south-west' of $\boldsymbol{x}_{1}$. The probability of this occurring is

$$
\left(1-\prod_{i=1}^{d} x_{i}\right)^{n-1}
$$

Hence,

$$
\begin{aligned}
\mathrm{E}\left(L_{n}\right) & =\sum_{i=1}^{n} \mathrm{E}\left(\left|\boldsymbol{x}_{i}\right| 1_{G_{i}}\right) \\
& =n \mathrm{E}\left(\left|\boldsymbol{x}_{1}\right| 1_{G_{1}}\right) \\
& =n \mathrm{E}\left(\left|\boldsymbol{x}_{1}\right|\right) \mathrm{E}\left(1_{G_{1}} \mid \boldsymbol{x}_{1}\right) \\
& =n \int_{0}^{1} \cdots \int_{0}^{1}\left(x_{1}^{2}+\cdots+x_{d}^{2}\right)^{1 / 2}\left(1-\prod_{i=1}^{d} x_{i}\right)^{n-1} \mathrm{~d} x_{1} \cdots \mathrm{d} x_{d} .
\end{aligned}
$$

To estimate the above integral, we use the following two elementary inequalities:

$$
\begin{gathered}
\left(\sum_{i=1}^{d} x_{i}\right)\left(1-\frac{\sum_{i \neq j} x_{i} x_{j}}{\left(\sum_{i=1}^{d} x_{i}\right)^{2}}\right) \leq\left(\sum_{i=1}^{d} x_{i}^{2}\right)^{1 / 2} \leq \sum_{i=1}^{d} x_{i}, \quad x_{i}>0, \\
\left(1-n x^{2}\right) \mathrm{e}^{-n x} \leq(1-x)^{n} \leq \mathrm{e}^{-n x} .
\end{gathered}
$$


By applying the second inequality of (9) to (8), we have

$$
\begin{aligned}
\mathrm{E}\left(L_{n}\right) & \leq n \int_{0}^{1} \cdots \int_{0}^{1}\left(x_{1}+\cdots+x_{d}\right)\left(1-\prod_{i=1}^{d} x_{i}\right)^{n-1} \mathrm{~d} x_{1} \cdots \mathrm{d} x_{d} \quad \text { (by symmetry) } \\
& =d n \int_{0}^{1} \cdots \int_{0}^{1} x_{1}\left(1-\prod_{i=1}^{d} x_{i}\right)^{n-1} \mathrm{~d} x_{1} \cdots \mathrm{d} x_{d} \\
& \left.\leq d n \int_{0}^{1} \cdots \int_{0}^{1} x_{1} \exp \left(-(n-1) \prod_{i=1}^{d} x_{i}\right) \mathrm{d} x_{1} \cdots \mathrm{d} x_{d} \quad \text { (by }(10)\right) \\
& =d n \int_{0}^{1} \mathrm{~d} x \int_{0}^{\infty} \cdots \int_{0}^{\infty} \mathrm{d} y_{2} \cdots \mathrm{d} y_{d} \\
& =d n \int_{0}^{1} \mathrm{~d} x \int_{0}^{\infty} x \mathrm{e}^{-(n-1) x \mathrm{e}^{-z}} \mathrm{e}^{-z} \frac{z^{d-2}}{(d-2) !} \mathrm{d} z \\
& =d \frac{n}{n-1} \int_{0}^{1} \mathrm{~d} x \int_{-\log (n-1) x}^{\infty} \mathrm{e}^{-\mathrm{e}^{-u}} \mathrm{e}^{-u} \frac{(u+\log (n-1) x)^{d-2}}{(d-2) !} \mathrm{d} u \\
& =d \frac{n}{n-1} \int_{0}^{1} \mathrm{~d} x \int_{0}^{(n-1) x} \mathrm{e}^{-v} \frac{(-\log v+\log (n-1) x)^{d-2}}{(d-2) !} \mathrm{d} v .
\end{aligned}
$$

Here we have made the following changes of variable, in the order listed:

- $x_{1}=: x$ and $x_{i}=: \mathrm{e}^{-y_{i}}, i=2, \ldots, d$,

- $\sum_{j=2}^{d} y_{j}=: z$,

- $z-\log (n-1) x=: u$,

- $\mathrm{e}^{-u}=: v$.

Now we expand the term

$$
(-\log v+\log (n-1) x)^{d-2}=(-\log v+\log (n-1)+\log x)^{d-2}
$$

and integrate term by term. We can then easily see that integration of the $(\log (n-1))^{d-2}$ term gives the leading term and that the other terms are all smaller than the leading term at least by a factor of $(\log n)^{-1}$. Hence, as $n \rightarrow \infty$,

$$
\begin{aligned}
\mathrm{E}\left(L_{n}\right) & \leq\left(d \frac{n}{n-1} \int_{0}^{1} \mathrm{~d} x \int_{0}^{(n-1) x} \mathrm{e}^{-v} \mathrm{~d} v+O\left((\log n)^{-1}\right)\right) \frac{(\log (n-1))^{d-2}}{(d-2) !} \\
& =\left(d \frac{n}{n-1} \int_{0}^{1} \mathrm{~d} x \int_{0}^{\infty} \mathrm{e}^{-v} \mathrm{~d} v+O\left(n^{-1}\right)+O\left((\log n)^{-1}\right)\right) \frac{(\log (n-1))^{d-2}}{(d-2) !} \\
& =\left(d \int_{0}^{1} \mathrm{~d} x \int_{0}^{\infty} \mathrm{e}^{-v} \mathrm{~d} v+O\left(n^{-1}\right)+O\left(n^{-1}\right)+O\left((\log n)^{-1}\right)\right) \frac{(\log (n-1))^{d-2}}{(d-2) !} \\
& \asymp \frac{d}{(d-2) !}(\log n)^{d-2} .
\end{aligned}
$$


Before we continue, we would like to point out that many integral calculations in Sections 2 and 3 follow a procedure very similar to that in (11) and (12); namely change $(1-a)^{b}$ to $\mathrm{e}^{-a b}$ using (10), change the product $\prod_{j=2}^{d} x_{j}$ to the sum $\exp \left(-\sum_{j=2}^{d} y_{j}\right)$ using a change of variable, use the hyperplane parameter $\sum_{j=2}^{d} y_{j}=: z$, modify the hyperplane parameter to simplify the exponent, expand the integrand, and find the leading term. Thus, we refer to integral calculations similar to those in (11) and (12) as the usual argument and sometimes denote the usual argument as '. . ' ' in equations.

Recall that, to obtain an asymptotic upper bound (12) of $\mathrm{E}\left(L_{n}\right)$, we used two elementary but useful inequalities: the second inequality of (9) and the second inequality of (10). The difference between $\mathrm{E}\left(L_{n}\right)$ and the asymptotic upper bound (12) thus consists of two parts: the error caused by the use of the second inequality of (10) and the error caused by the use of the second inequality of (9).

By the usual argument, we see that the error caused by the use of the second inequality of (10) is bounded by

$$
\begin{aligned}
& d n(n-1) \int_{0}^{1} \cdots \int_{0}^{1} x_{1} \prod_{i=1}^{d} x_{i}^{2} \exp \left(-(n-1) \prod_{i=1}^{d} x_{i}\right) \mathrm{d} x_{1} \cdots \mathrm{d} x_{d} \\
& =O\left(n^{-1}(\log n)^{d-2}\right)
\end{aligned}
$$

(using the difference between the upper and lower bounds of (10)).

Again by the usual argument, we also see that the error caused by the use of the second inequality of (9) is bounded by

$$
\begin{aligned}
n \int_{0}^{1} & \cdots \int_{0}^{1} \frac{\sum_{i \neq j} x_{i} x_{j}}{\sum_{i=1}^{d} x_{i}}\left(1-\prod_{i=1}^{d} x_{i}\right)^{n-1} \mathrm{~d} x_{1} \cdots \mathrm{d} x_{d} \\
& \leq n \int_{0}^{1} \cdots \int_{0}^{1} \frac{\sum_{i \neq j} x_{i} x_{j}}{\sum_{i=1}^{d} x_{i}} \exp \left(-(n-1) \prod_{i=1}^{d} x_{i}\right) \mathrm{d} x_{1} \cdots \mathrm{d} x_{d} \quad(\text { by }(10)) \\
& =d(d-1) n \int_{0}^{1} \cdots \int_{0}^{1} \frac{x_{1} x_{2}}{\sum_{i=1}^{d} x_{i}} \exp \left(-(n-1) \prod_{i=1}^{d} x_{i}\right) \mathrm{d} x_{1} \cdots \mathrm{d} x_{d} \quad \text { (by symmetry) } \\
& \leq d(d-1) n \int_{0}^{1} \cdots \int_{0}^{1} \frac{x_{1} x_{2}}{x_{1}+x_{2}} \exp \left(-(n-1) \prod_{i=1}^{d} x_{i}\right) \mathrm{d} x_{1} \cdots \mathrm{d} x_{d} \\
& \leq d(d-1) n \int_{0}^{1} \cdots \int_{0}^{1} \sqrt{x_{1} x_{2}} \exp \left(-(n-1) \prod_{i=1}^{d} x_{i}\right) \mathrm{d} x_{1} \cdots \mathrm{d} x_{d}
\end{aligned}
$$

(by the AM-GM-HM inequality)

$$
=O\left((\log n)^{d-3}\right)
$$

(using the difference between the upper and lower bounds of (9)). Therefore, Theorem 1 follows from (12)-(14). 


\section{Variance}

In this section we prove Theorem 2. The basic idea of the proof of Theorem 2 is the same as that of Theorem 1. Using (15) we write $\operatorname{var}\left(L_{n}\right)$ as exact integrals. Using inequalities (9) and (10) we approximate the exact integrals by more tractable integrals. Then, by evaluating these tractable integrals, we recover Theorem 2. Compared to the proof of Theorem 1, in the proof of Theorem 2 there are more complicated integrals. However, the basic idea of the evaluation of the integrals is the same: we use the usual argument.

We start with an obvious observation; by (7),

$$
\begin{aligned}
\operatorname{var}\left(L_{n}\right) & =\sum_{i=1}^{n} \operatorname{var}\left(\left|\boldsymbol{x}_{i}\right| 1_{G_{i}}\right)+\sum_{i \neq j} \operatorname{cov}\left(\left|\boldsymbol{x}_{i}\right| 1_{G_{i}},\left|\boldsymbol{x}_{j}\right| 1_{G_{j}}\right) \\
& =n \operatorname{var}\left(\left|\boldsymbol{x}_{1}\right| 1_{G_{1}}\right)+n(n-1) \operatorname{cov}\left(\left|\boldsymbol{x}_{1}\right| 1_{G_{1}},\left|\boldsymbol{x}_{2}\right| 1_{G_{2}}\right) \\
& =n \operatorname{var}\left(\left|\boldsymbol{x}_{1}\right| 1_{G_{1}}\right)+n^{2}\left(1+O\left(n^{-1}\right)\right) \operatorname{cov}\left(\left|\boldsymbol{x}_{1}\right| 1_{G_{1}},\left|\boldsymbol{x}_{2}\right| 1_{G_{2}}\right) .
\end{aligned}
$$

Since

$$
n \operatorname{var}\left(\left|\boldsymbol{x}_{1}\right| 1_{G_{1}}\right)=n\left(\mathrm{E}\left(\left|\boldsymbol{x}_{1}\right|^{2} 1_{G_{1}}\right)-\left[\mathrm{E}\left(\left|\boldsymbol{x}_{1}\right| 1_{G_{1}}\right)\right]^{2}\right),
$$

we estimate $n \mathrm{E}\left(\left|x_{1}\right|^{2} 1_{G_{1}}\right)$ first. By the usual argument, calculations similar to (11) and (12) yield

$$
\begin{aligned}
n \mathrm{E}\left(\left|\boldsymbol{x}_{1}\right|^{2} 1_{G_{1}}\right) & =n \int_{0}^{1} \cdots \int_{0}^{1}\left(x_{1}^{2}+\cdots+x_{d}^{2}\right)\left(1-\prod_{i=1}^{d} x_{i}\right)^{n-1} \mathrm{~d} x_{1} \cdots \mathrm{d} x_{d} \\
& =d n \int_{0}^{1} \cdots \int_{0}^{1} x_{1}^{2}\left(1-\prod_{i=1}^{d} x_{i}\right)^{n-1} \mathrm{~d} x_{1} \cdots \mathrm{d} x_{d} \\
& \vdots \\
& \asymp \frac{1}{2} \frac{d}{(d-2) !}(\log n)^{d-2} .
\end{aligned}
$$

Thus, by (16) and (2),

$$
\begin{aligned}
n \operatorname{var}\left(\left|\boldsymbol{x}_{1}\right| 1_{G_{1}}\right) & =n \mathrm{E}\left(\left|\boldsymbol{x}_{1}\right|^{2} 1_{G_{1}}\right)-n\left[\mathrm{E}\left(\left|\boldsymbol{x}_{1}\right| 1_{G_{1}}\right)\right]^{2} \\
& =n \mathrm{E}\left(\left|\boldsymbol{x}_{1}\right|^{2} 1_{G_{1}}\right)-n\left(\frac{\mathrm{E}\left(L_{n}\right)}{n}\right)^{2} \\
& \asymp \frac{1}{2} \frac{d}{(d-2) !}(\log n)^{d-2} .
\end{aligned}
$$

Now let us look at the crossing term

$$
n^{2}\left(1+O\left(n^{-1}\right)\right) \operatorname{cov}\left(\left|\boldsymbol{x}_{1}\right| 1_{G_{1}},\left|\boldsymbol{x}_{2}\right| 1_{G_{2}}\right)
$$

Let us say that $\boldsymbol{x}$ dominates $\boldsymbol{y}$ if $\boldsymbol{y} \prec \boldsymbol{x}$, and let

$$
D=\left\{(\boldsymbol{x}, \boldsymbol{y}) \in[0,1]^{d} \times[0,1]^{d}: \boldsymbol{x} \text { does not dominate } \boldsymbol{y} \text { and } \boldsymbol{y} \text { does not dominate } \boldsymbol{x}\right\} .
$$


Then by symmetry, with the notation $\boldsymbol{x}=\left(x_{1}, \ldots, x_{d}\right)$ and $\boldsymbol{y}=\left(y_{1}, \ldots, y_{d}\right)$,

$$
\begin{aligned}
n^{2} \operatorname{cov}\left(\left|\boldsymbol{x}_{1}\right| 1_{G_{1}},\left|\boldsymbol{x}_{2}\right| 1_{G_{2}}\right) & \\
= & n^{2}\left(\int_{D}|\boldsymbol{x}||\boldsymbol{y}|\left(1-\prod_{i=1}^{d} x_{i}-\prod_{i=1}^{d} y_{i}+\prod_{i=1}^{d}\left(x_{i} \wedge y_{i}\right)\right)^{n-2} \mathrm{~d} \boldsymbol{x} \mathrm{d} \boldsymbol{y}\right. \\
& \left.\quad-\int_{[0,1]^{d} \times[0,1]^{d}}|\boldsymbol{x}||\boldsymbol{y}|\left(1-\prod_{i=1}^{d} x_{i}\right)^{n-1}\left(1-\prod_{i=1}^{d} y_{i}\right)^{n-1} \mathrm{~d} \boldsymbol{x} \mathrm{d} \boldsymbol{y}\right) \\
= & n^{2}\left(\int_{D}|\boldsymbol{x}||\boldsymbol{y}| f(\boldsymbol{x}, \boldsymbol{y}) \mathrm{d} \boldsymbol{x} \mathrm{d} \boldsymbol{y}\right. \\
& \left.\quad-2 \int_{\boldsymbol{x} \prec \boldsymbol{y}}|\boldsymbol{x}||\boldsymbol{y}|\left(1-\prod_{i=1}^{d} x_{i}\right)^{n-1}\left(1-\prod_{i=1}^{d} y_{i}\right)^{n-1} \mathrm{~d} \boldsymbol{x} \mathrm{d} \boldsymbol{y}\right) \\
= & : I_{1}-I_{2},
\end{aligned}
$$

where

$$
f(\boldsymbol{x}, \boldsymbol{y})=\left(1-\prod_{i=1}^{d} x_{i}-\prod_{i=1}^{d} y_{i}+\prod_{i=1}^{d}\left(x_{i} \wedge y_{i}\right)\right)^{n-2}-\left(1-\prod_{i=1}^{d} x_{i}\right)^{n-1}\left(1-\prod_{i=1}^{d} y_{i}\right)^{n-1} .
$$

Since

$$
1-\prod_{i=1}^{d} x_{i}-\prod_{i=1}^{d} y_{i}+\prod_{i=1}^{d}\left(x_{i} \wedge y_{i}\right) \geq\left(1-\prod_{i=1}^{d} x_{i}\right)\left(1-\prod_{i=1}^{d} y_{i}\right),
$$

we have $f(\boldsymbol{x}, \boldsymbol{y}) \geq 0$ and, hence, $I_{1} \geq 0$. To obtain the asymptotics of $I_{1}$, we decompose $D$ according to the number, $k$, of the components of $\boldsymbol{x}$ that are larger than the corresponding components of $\boldsymbol{y}$. Then, where in an obvious change of variable we write $x_{i}$ as a large component of $\boldsymbol{x}$ or $\boldsymbol{y}$ and $x_{i} u_{i}$ as a small component of $\boldsymbol{x}$ or $\boldsymbol{y}$, we have

$$
\begin{aligned}
I_{1}=n^{2} & \sum_{k=1}^{d-1}\left(\begin{array}{l}
d \\
k
\end{array}\right) \int_{[0,1]^{d} \times[0,1]^{d}}\left(\sum_{i=1}^{k} x_{i}^{2}+\sum_{i=k+1}^{d} x_{i}^{2} u_{i}^{2}\right)^{1 / 2}\left(\sum_{i=1}^{k} x_{i}^{2} u_{i}^{2}+\sum_{i=k+1}^{d} x_{i}^{2}\right)^{1 / 2} \\
& \times\left(\left(1-\prod_{i=1}^{d} x_{i} \prod_{j=k+1}^{d} u_{j}-\prod_{i=1}^{d} x_{i} \prod_{j=1}^{k} u_{j}+\prod_{i=1}^{d} x_{i} \prod_{j=1}^{d} u_{j}\right)^{n-2}\right. \\
& \left.-\left(1-\prod_{i=1}^{d} x_{i} \prod_{j=k+1}^{d} u_{j}\right)^{n-1}\left(1-\prod_{i=1}^{d} x_{i} \prod_{j=1}^{k} u_{j}\right)^{n-1}\right) \prod_{i=1}^{d} x_{i} \mathrm{~d} \boldsymbol{x} \mathrm{d} \boldsymbol{u} .
\end{aligned}
$$

We replace the product terms by exponential terms as we did in (11). With these replacements there will be two errors of order $n^{-1 / 2}(\log n)^{d-1}$. Using the difference between the upper and lower bounds of (10), we see that the error caused by the replacement of

$$
\left(1-\prod_{i=1}^{d} x_{i} \prod_{j=k+1}^{d} u_{j}-\prod_{i=1}^{d} x_{i} \prod_{j=1}^{k} u_{j}+\prod_{i=1}^{d} x_{i} \prod_{j=1}^{d} u_{j}\right)^{n-2}
$$


is bounded by

$$
\begin{aligned}
C n^{3} \int_{[0,1]^{d} \times[0,1]^{d}}\left(\prod_{i=1}^{d} x_{i}\right)^{3}\left(\prod_{j=k+1}^{d} u_{j}+\prod_{j=1}^{k} u_{j}-\prod_{j=1}^{d} u_{j}\right)^{2} \\
\quad \times \exp \left(-(n-2) \prod_{i=1}^{d} x_{i}\left(\prod_{j=k+1}^{d} u_{j}+\prod_{j=1}^{k} u_{j}-\prod_{j=1}^{d} u_{j}\right)\right) \mathrm{d} \boldsymbol{x} \mathrm{d} \boldsymbol{u} \\
\leq \quad C n^{3} \int_{[0,1]^{d} \times[0,1]^{d}}\left(\prod_{i=1}^{d} x_{i}\right)^{5 / 2}\left(\prod_{j=k+1}^{d} u_{j}+\prod_{j=1}^{k} u_{j}-\prod_{j=1}^{d} u_{j}\right)^{2} \\
\quad \times \exp \left(-(n-2) \prod_{i=1}^{d} x_{i}\left(\prod_{j=k+1}^{d} u_{j}+\prod_{j=1}^{k} u_{j}-\prod_{j=1}^{d} u_{j}\right)\right) \mathrm{d} \boldsymbol{x} \mathrm{d} \boldsymbol{u} \\
\vdots \quad \int_{[0,1]^{d}}\left(\prod_{j=k+1}^{d} u_{j}+\prod_{j=1}^{k} u_{j}-\prod_{j=1}^{d} u_{j}\right)^{-3 / 2} \mathrm{~d} \boldsymbol{u} \\
\leq \quad C n^{-1 / 2}(\log n)^{d-1} \int_{\left(n^{-1 / 2}(\log n)^{d-1}\right)}
\end{aligned}
$$

(since $x^{3} \leq x^{5 / 2}$ for $0 \leq x \leq 1$ ). Here, we have used the fact that

$$
\begin{aligned}
& \int_{[0,1]^{d}}\left(\prod_{j=k+1}^{d} u_{j}+\prod_{j=1}^{k} u_{j}-\prod_{j=1}^{d} u_{j}\right)^{-3 / 2} \mathrm{~d} \boldsymbol{u} \\
& \quad \leq \int_{[0,1]^{d}}\left(2\left(\prod_{j=1}^{d} u_{j}\right)^{1 / 2}-\prod_{j=1}^{d} u_{j}\right)^{-3 / 2} \mathrm{~d} \boldsymbol{u} \quad(\text { since } x+y \geq 2 \sqrt{x y} \text { for } x, y>0) \\
& \quad \leq \int_{[0,1]^{d}}\left(\prod_{j=1}^{d} u_{j}\right)^{-3 / 4} \mathrm{~d} \boldsymbol{u}<\infty \quad(\text { since } x \leq \sqrt{x} \text { for } 0 \leq x \leq 1) .
\end{aligned}
$$

Because

$$
\begin{aligned}
& \int_{[0,1]^{d}} \frac{\left(\prod_{j=k+1}^{d} u_{j}\right)^{2}}{\left(\prod_{j=k+1}^{d} u_{j}+\prod_{j=1}^{k} u_{j}\right)^{7 / 2}} \mathrm{~d} \boldsymbol{u} \\
& \quad \leq C \int_{[0,1]^{d}}\left(\prod_{j=k+1}^{d} u_{j}\right)^{-5 / 8}\left(\prod_{j=1}^{k} u_{j}\right)^{-7 / 8} \mathrm{~d} \boldsymbol{u} \\
& \quad<\infty
\end{aligned}
$$

(since $(x+y)^{4} \geq 4 x y^{3}$ for $\left.x, y>0\right)$ and

$\int_{[0,1]^{d}} \frac{\left(\prod_{j=1}^{k} u_{j}\right)^{2}}{\left(\prod_{j=k+1}^{d} u_{j}+\prod_{j=1}^{k} u_{j}\right)^{7 / 2}} \mathrm{~d} \boldsymbol{u}<\infty \quad$ (by the argument of (20)), 
by the argument of (19) we see also that the error caused by the replacement of

$$
\left(1-\prod_{i=1}^{d} x_{i} \prod_{j=k+1}^{d} u_{j}\right)^{n-1}\left(1-\prod_{i=1}^{d} x_{i} \prod_{j=1}^{k} u_{j}\right)^{n-1}
$$

is of order $n^{-1 / 2}(\log n)^{d-1}$.

We further replace the factors $(n-2)$ and $(n-1)$ by $n$ in the corresponding exponents; as we see in (11) and (12), this does not alter the leading term. We then replace

$$
\left(\sum_{i=1}^{k} x_{i}^{2}+\sum_{i=k+1}^{d} x_{i}^{2} u_{i}^{2}\right)^{1 / 2} \text { and }\left(\sum_{i=1}^{k} x_{i}^{2} u_{i}^{2}+\sum_{i=k+1}^{d} x_{i}^{2}\right)^{1 / 2}
$$

by

$$
\left(\sum_{i=1}^{k} x_{i}+\sum_{i=k+1}^{d} x_{i} u_{i}\right) \text { and }\left(\sum_{i=1}^{k} x_{i} u_{i}+\sum_{i=k+1}^{d} x_{i}\right),
$$

respectively. With this replacement there will be an error $a_{n}$. Thus,

$$
\begin{aligned}
& I_{1}=n^{2} \sum_{k=1}^{d-1}\left(\begin{array}{l}
d \\
k
\end{array}\right) \int_{[0,1]^{d} \times[0,1]^{d}}\left(\sum_{i=1}^{k} x_{i}+\sum_{i=k+1}^{d} x_{i} u_{i}\right)\left(\sum_{i=1}^{k} x_{i} u_{i}+\sum_{i=k+1}^{d} x_{i}\right) \\
& \times \exp \left(-n \prod_{i=1}^{d} x_{i}\left(\prod_{j=k+1}^{d} u_{j}+\prod_{j=1}^{k} u_{j}\right)\right) \\
& \times\left(\exp \left(n \prod_{i=1}^{d} x_{i} \prod_{j=1}^{d} u_{j}\right)-1\right) \prod_{i=1}^{d} x_{i} \mathrm{~d} \boldsymbol{x} \mathrm{d} \boldsymbol{u} \\
& +O\left(n^{-1 / 2}(\log n)^{d-1}\right)+a_{n} \\
& =n^{2} \sum_{k=1}^{d-1}\left(\begin{array}{l}
d \\
k
\end{array}\right) \int_{[0,1]^{d} \times[0,1]^{d}}\left[k x_{1}^{2} u_{1}+k(k-1) x_{1} u_{1} x_{2}+k(d-k) x_{1} x_{d}+(d-k) x_{d}^{2} u_{d}\right. \\
& \left.+(d-k)(d-k-1) x_{d} u_{d} x_{d-1}+(d-k) k x_{1} u_{1} x_{d} u_{d}\right] \\
& \times \exp \left(-n \prod_{i=1}^{d} x_{i}\left(\prod_{j=k+1}^{d} u_{j}+\prod_{j=1}^{k} u_{j}\right)\right) \\
& \times\left(\exp \left(n \prod_{i=1}^{d} x_{i} \prod_{j=1}^{d} u_{j}\right)-1\right) \\
& \times \prod_{i=1}^{d} x_{i} \mathrm{~d} \boldsymbol{x} \mathrm{d} \boldsymbol{u} \\
& +O\left(n^{-1 / 2}(\log n)^{d-1}\right)+a_{n} \\
& =: H_{1}+H_{2}+H_{3}+H_{4}+H_{5}+H_{6}+O\left(n^{-1 / 2}(\log n)^{d-1}\right)+a_{n} \text {. }
\end{aligned}
$$


We first look at the terms with $k \geq 2$ in $H_{1}$. Starting with the obvious changes of variable

$$
x_{i}=: \mathrm{e}^{-y_{i}} \quad \text { and } \quad u_{i}=: \mathrm{e}^{-v_{i}}, \quad 2 \leq i \leq d,
$$

and

$$
\begin{gathered}
\sum_{i=2}^{d} y_{i}=: z, \\
\sum_{j=2}^{k} v_{j}=: w_{1}, \\
\sum_{j=k+1}^{d} v_{j}=: w_{2},
\end{gathered}
$$

by the usual argument we obtain

$$
\begin{aligned}
& n^{2} \int_{[0,1]^{d} \times[0,1]^{d}} x_{1}^{2} u_{1} \exp \left(-n \prod_{i=1}^{d} x_{i}\left(\prod_{j=k+1}^{d} u_{j}+\prod_{j=1}^{k} u_{j}\right)\right) \\
& \times\left(\exp \left(n \prod_{i=1}^{d} x_{i} \prod_{j=1}^{d} u_{j}\right)-1\right) \prod_{i=1}^{d} x_{i} \mathrm{~d} \boldsymbol{x} \mathrm{d} \boldsymbol{u} \\
& \vdots \\
& =\int_{0}^{1} \mathrm{~d} x_{1} \int_{0}^{1} \mathrm{~d} u_{1} \int_{0}^{n x_{1}} \mathrm{~d} z \int_{0}^{u_{1}} \mathrm{~d} w_{1} \int_{0}^{1} \mathrm{~d} w_{2} x_{1} \mathrm{e}^{-z w_{2}-z w_{1}}\left(\mathrm{e}^{z w_{1} w_{2}}-1\right) z \\
& \times \frac{\left(-\log z+\log n x_{1}\right)^{d-2}}{(d-2) !} \frac{\left(-\log w_{1}+\log u_{1}\right)^{k-2}}{(k-2) !} \frac{\left(-\log w_{2}\right)^{d-k-1}}{(d-k-1) !} \\
& =: \int_{0}^{1} \mathrm{~d} x_{1} \int_{0}^{1} \mathrm{~d} u_{1} \int_{0}^{\infty} \mathrm{d} z \int_{0}^{u_{1}} \mathrm{~d} w_{1} \int_{0}^{1} \mathrm{~d} w_{2} x_{1} \mathrm{e}^{-z w_{2}-z w_{1}}\left(\mathrm{e}^{z w_{1} w_{2}}-1\right) z \\
& \times \frac{\left(-\log z+\log n x_{1}\right)^{d-2}}{(d-2) !} \frac{\left(-\log w_{1}+\log u_{1}\right)^{k-2}}{(k-2) !} \frac{\left(-\log w_{2}\right)^{d-k-1}}{(d-k-1) !}+b_{n} \\
& \asymp \int_{0}^{1} \mathrm{~d} x_{1} \int_{0}^{1} \mathrm{~d} u_{1} \int_{0}^{\infty} \mathrm{d} z \int_{0}^{u_{1}} \mathrm{~d} w_{1} \int_{0}^{1} \mathrm{~d} w_{2} x_{1} \mathrm{e}^{-z w_{2}-z w_{1}}\left(\mathrm{e}^{z w_{1} w_{2}}-1\right) z \\
& \times \frac{(\log n)^{d-2}}{(d-2) !} \frac{\left(-\log w_{1}+\log u_{1}\right)^{k-2}}{(k-2) !} \frac{\left(-\log w_{2}\right)^{d-k-1}}{(d-k-1) !}+b_{n} \\
& =\int_{0}^{1} \mathrm{~d} u_{1} \int_{0}^{u_{1}} \mathrm{~d} w_{1} \int_{0}^{1} \mathrm{~d} w_{2}\left(\left(w_{1}+w_{2}-w_{1} w_{2}\right)^{-2}-\left(w_{1}+w_{2}\right)^{-2}\right) \\
& \times \frac{1}{2} \frac{(\log n)^{d-2}}{(d-2) !} \frac{\left(-\log w_{1}+\log u_{1}\right)^{k-2}}{(k-2) !} \frac{\left(-\log w_{2}\right)^{d-k-1}}{(d-k-1) !}+b_{n} \\
& =: h_{k}(\log n)^{d-2}+b_{n} \\
& \asymp h_{k}(\log n)^{d-2} \quad\left(\text { since } b_{n}=O\left((\log n)^{d-2} n^{-1+\varepsilon}\right)\right. \text { by (25), below). }
\end{aligned}
$$

Now, let us check that

$$
b_{n}=O\left((\log n)^{d-2} n^{-1+\varepsilon}\right) .
$$


For $0 \leq w_{1}, w_{2} \leq 1$ and very small but strictly positive $\varepsilon$, we have

$$
\begin{gathered}
1-\left(1-w_{1}\right)^{1-\varepsilon} \leq w_{1}\left(1-w_{1}\right)^{-\varepsilon} \\
w_{1} \leq w_{1}+w_{2}-w_{1} w_{2}, \quad w_{2} \leq w_{1}+w_{2}-w_{1} w_{2}, \\
\left(w_{1}+w_{2}\right)^{-1} \leq\left(w_{1}+w_{2}-w_{1} w_{2}\right)^{-1} .
\end{gathered}
$$

Therefore,

$$
\begin{aligned}
& \left|b_{n}\right|=\mid \int_{0}^{1} \mathrm{~d} x_{1} \int_{0}^{1} \mathrm{~d} u_{1} \int_{n x_{1}}^{\infty} \mathrm{d} z \int_{0}^{u_{1}} \mathrm{~d} w_{1} \int_{0}^{1} \mathrm{~d} w_{2} x_{1} \mathrm{e}^{-z w_{2}-z w_{1}}\left(\mathrm{e}^{z w_{1} w_{2}}-1\right) z \\
& \times \frac{\left(-\log z+\log n x_{1}\right)^{d-2}}{(d-2) !} \frac{\left(-\log w_{1}+\log u_{1}\right)^{k-2}}{(k-2) !} \frac{\left(-\log w_{2}\right)^{d-k-1}}{(d-k-1) !} \mid \\
& \leq C(\log n)^{d-2} \int_{0}^{1} \mathrm{~d} x_{1} \int_{0}^{1} \mathrm{~d} u_{1} \int_{n x_{1}}^{\infty} \mathrm{d} z \int_{0}^{u_{1}} \mathrm{~d} w_{1} \int_{0}^{1} \mathrm{~d} w_{2} \\
& \times \mathrm{e}^{-z w_{2}-z w_{1}}\left(\mathrm{e}^{z w_{1} w_{2}}-1\right) x_{1} z\left(\frac{z}{x_{1}}\right)^{\varepsilon} w_{1}^{-\varepsilon} u_{1}^{-\varepsilon} w_{2}^{-\varepsilon} \\
& \leq C(\log n)^{d-2} \int_{0}^{1} \mathrm{~d} u_{1} \int_{0}^{u_{1}} \mathrm{~d} w_{1} \int_{0}^{\infty}\left(\min \left(\frac{z}{n}, 1\right)\right)^{1-\varepsilon} \mathrm{d} z \int_{0}^{1} \mathrm{~d} w_{2} \\
& \times \mathrm{e}^{-z w_{2}-z w_{1}}\left(\mathrm{e}^{z w_{1} w_{2}}-1\right) z^{1+\varepsilon} w_{1}^{-\varepsilon} u_{1}^{-\varepsilon} w_{2}^{-\varepsilon} \\
& \leq C(\log n)^{d-2} n^{-1+\varepsilon} \int_{0}^{1} \mathrm{~d} u_{1} \int_{0}^{u_{1}} \mathrm{~d} w_{1} \int_{0}^{\infty} \mathrm{d} z \int_{0}^{1} \mathrm{~d} w_{2} \\
& \times \mathrm{e}^{-z w_{2}-z w_{1}}\left(\mathrm{e}^{z w_{1} w_{2}}-1\right) z^{2} w_{1}^{-\varepsilon} u_{1}^{-\varepsilon} w_{2}^{-\varepsilon} \\
& =C(\log n)^{d-2} n^{-1+\varepsilon} \int_{0}^{1} \mathrm{~d} u_{1} \int_{0}^{u_{1}} \mathrm{~d} w_{1} \int_{0}^{1} \mathrm{~d} w_{2} \\
& \times\left(\frac{1}{\left(w_{1}+w_{2}-w_{1} w_{2}\right)^{3}}-\frac{1}{\left(w_{1}+w_{2}\right)^{3}}\right) w_{1}^{-\varepsilon} u_{1}^{-\varepsilon} w_{2}^{-\varepsilon} \\
& =C(\log n)^{d-2} n^{-1+\varepsilon} \int_{0}^{1} \mathrm{~d} w_{1} \int_{0}^{1} \mathrm{~d} w_{2}\left(1-\left(1-w_{1}\right)^{1-\varepsilon}\right) \\
& \times\left(\frac{1}{\left(w_{1}+w_{2}-w_{1} w_{2}\right)^{3}}-\frac{1}{\left(w_{1}+w_{2}\right)^{3}}\right) w_{1}^{-\varepsilon} w_{2}^{-\varepsilon} \\
& \leq C(\log n)^{d-2} n^{-1+\varepsilon} \int_{0}^{1} \mathrm{~d} w_{1} \int_{0}^{1} \mathrm{~d} w_{2} \frac{w_{1}^{2-2 \varepsilon} w_{2}^{1-\varepsilon}\left(1-w_{1}\right)^{-\varepsilon}}{\left(w_{1}+w_{2}-w_{1} w_{2}\right)^{3}\left(w_{1}+w_{2}\right)} \\
& \leq C(\log n)^{d-2} n^{-1+\varepsilon} \int_{0}^{1} \mathrm{~d} w_{1} \int_{0}^{1} \mathrm{~d} w_{2} \frac{1}{\left(1-w_{1}\right)^{\varepsilon}\left(w_{1}+w_{2}-w_{1} w_{2}\right)^{1+3 \varepsilon}} \\
& =C(\log n)^{d-2} n^{-1+\varepsilon} \int_{0}^{1} \frac{1}{\left(1-w_{1}\right)^{1+\varepsilon}}\left(w_{1}^{-3 \varepsilon}-1\right) \mathrm{d} w_{1} \\
& =O\left((\log n)^{d-2} n^{-1+\varepsilon}\right) \text {. }
\end{aligned}
$$

Now we consider the term with $k=1$ in $H_{1}$. From the above calculation, we see that in this case there is no need to make the change of variable $\sum_{j=2}^{k} v_{j}=: w_{1}$. With this in mind we 
just follow the above calculation, to obtain

$$
\begin{aligned}
& n^{2} \int_{[0,1]^{d} \times[0,1]^{d}} x_{1}^{2} u_{1} \exp \left(-n \prod_{i=1}^{d} x_{i}\left(\prod_{j=2}^{d} u_{j}+u_{1}\right)\right) \\
& \quad \times\left(\exp \left(n \prod_{i=1}^{d} x_{i} \prod_{j=1}^{d} u_{j}\right)-1\right) \prod_{i=1}^{d} x_{i} \mathrm{~d} \boldsymbol{x} \mathrm{d} \boldsymbol{u} \\
& \asymp \int_{0}^{1} \mathrm{~d} u_{1} \int_{0}^{1} \mathrm{~d} w_{2} u_{1}\left(\left(u_{1}+w_{2}-u_{1} w_{2}\right)^{-2}-\left(u_{1}+w_{2}\right)^{-2}\right) \\
& \quad \times \frac{1}{2} \frac{(\log n)^{d-2}}{(d-2) !} \frac{\left(-\log w_{2}\right)^{d-2}}{(d-2) !} \\
& =h_{1}(\log n)^{d-2}, \quad
\end{aligned}
$$

and, hence, by (26) and (22),

$$
H_{1} \asymp \sum_{k=1}^{d-1}\left(\begin{array}{l}
d \\
k
\end{array}\right) k h_{k}(\log n)^{d-2},
$$

where the $h_{k}$ are given by (4) and (5). By similar, but somewhat simpler, calculations, we obtain

$$
H_{2}=O\left((\log n)^{d-3}\right)
$$

and

$$
H_{3}=O\left((\log n)^{d-3}\right)
$$

By symmetry, we then have

$$
H_{4}=H_{1}, \quad H_{5}=H_{2}, \quad H_{6}=H_{3} .
$$

Therefore, by (21) and (27)-(30),

$$
\begin{aligned}
I_{1} & \asymp 2 \sum_{k=1}^{d-1}\left(\begin{array}{l}
d \\
k
\end{array}\right) k h_{k}(\log n)^{d-2}+O\left(n^{-1 / 2}(\log n)^{d-1}\right)+a_{n} \\
& \asymp 2 \sum_{k=1}^{d-1}\left(\begin{array}{l}
d \\
k
\end{array}\right) k h_{k}(\log n)^{d-2}+a_{n} .
\end{aligned}
$$

Next we consider $a_{n}$. Since we will see a very similar calculation for $I_{2}$ in great detail below, here we just sketch how to estimate the error term $a_{n}$. By the argument used in the proof of (14), we see that

$$
\begin{aligned}
a_{n} \leq C n^{2} \sum_{k=1}^{d-1}\left(\begin{array}{l}
d \\
k
\end{array}\right) \int_{[0,1]^{d} \times[0,1]^{d}}\left(\left(x_{1} x_{2}\right)^{1 / 2}+\left(x_{1} x_{d} u_{d}\right)^{1 / 2}+\left(x_{d-1} u_{d-1} x_{d} u_{d}\right)^{1 / 2}\right) \\
\quad \times\left(\sum_{i=1}^{k} x_{i} u_{i}+\sum_{i=k+1}^{d} x_{i}\right) \exp \left(-n \prod_{i=1}^{d} x_{i}\left(\prod_{j=k+1}^{d} u_{j}+\prod_{j=1}^{k} u_{j}\right)\right) \\
\times\left(\exp \left(n \prod_{i=1}^{d} x_{i} \prod_{j=1}^{d} u_{j}\right)-1\right) \prod_{i=1}^{d} x_{i} \mathrm{~d} \boldsymbol{x} \mathrm{d} \boldsymbol{u} .
\end{aligned}
$$


Now we just follow the argument for (22)-(31). Since each term in the expansion of

$$
\left(\left(x_{1} x_{2}\right)^{1 / 2}+\left(x_{1} x_{d} u_{d}\right)^{1 / 2}+\left(x_{d-1} u_{d-1} x_{d} u_{d}\right)^{1 / 2}\right)\left(\sum_{i=1}^{k} x_{i} u_{i}+\sum_{i=k+1}^{d} x_{i}\right)
$$

has at least two different $x_{j}$, by the argument for (22)-(31) we see that each integral in (32) is of order $(\log n)^{d-3}$, and that

$$
a_{n}=O\left((\log n)^{d-3}\right) .
$$

Let us now consider

$$
I_{2}=2 n^{2} \int_{\boldsymbol{x} \prec \boldsymbol{y}}|\boldsymbol{x}||\boldsymbol{y}|\left(1-\prod_{i=1}^{d} x_{i}\right)^{n-1}\left(1-\prod_{i=1}^{d} y_{i}\right)^{n-1} \mathrm{~d} \boldsymbol{x} \mathrm{d} \boldsymbol{y} .
$$

We make the following changes of variable: $x_{i}=: u_{i} v_{i}$ and $y_{i}=: u_{i}, i=1, \ldots, d$. We also replace

$$
\left(1-\prod_{i=1}^{d} x_{i}\right)^{n-1} \text { and }\left(1-\prod_{i=1}^{d} y_{i}\right)^{n-1}
$$

by

$$
\exp \left(-n \prod_{i=1}^{d} x_{i}\right) \text { and } \exp \left(-n \prod_{i=1}^{d} y_{i}\right)
$$

respectively. As we saw in (11), this approximation is valid. Also, by using (10) we replace $\left(\sum_{i=1}^{d} u_{i}^{2} v_{i}^{2}\right)^{1 / 2}\left(\sum_{i=1}^{d} u_{i}^{2}\right)^{1 / 2}$ by $\left(\sum_{i=1}^{d} u_{i} v_{i}\right)\left(\sum_{j=1}^{d} u_{j}\right)$. Thus, we have

$$
\begin{aligned}
& I_{2} \asymp 2 n^{2} \int_{[0,1]^{d} \times[0,1]^{d}} \sum_{i=1}^{d} \sum_{j=1}^{d} u_{i} v_{i} u_{j} \exp \left(-n \prod_{i=1}^{d} u_{i}\left(1+\prod_{i=1}^{d} v_{i}\right)\right) \prod_{i=1}^{d} u_{i} \mathrm{~d} \boldsymbol{u} \mathrm{d} \boldsymbol{v} \\
& =2 d n^{2} \int_{[0,1]^{d} \times[0,1]^{d}} u_{1}^{2} v_{1} \exp \left(-n \prod_{i=1}^{d} u_{i}\left(1+\prod_{i=1}^{d} v_{i}\right)\right) \prod_{i=1}^{d} u_{i} \mathrm{~d} \boldsymbol{u} \mathrm{d} \boldsymbol{v} \\
& \quad+2 d(d-1) n^{2} \int_{[0,1]^{d} \times[0,1]^{d}} u_{1} v_{1} u_{2} \exp \left(-n \prod_{i=1}^{d} u_{i}\left(1+\prod_{i=1}^{d} v_{i}\right)\right) \prod_{i=1}^{d} u_{i} \mathrm{~d} \boldsymbol{u} \mathrm{d} \boldsymbol{v} \\
& =: J_{1}+J_{2} .
\end{aligned}
$$

We first consider $J_{2}$, the simpler term:

$$
\begin{aligned}
J_{2} & =2 d(d-1) n^{2} \int_{[0,1]^{d} \times[0,1]^{d}} u_{1} v_{1} u_{2} \exp \left(-n \prod_{i=1}^{d} u_{i}\left(1+\prod_{i=1}^{d} v_{i}\right)\right) \prod_{i=1}^{d} u_{i} \mathrm{~d} \boldsymbol{u} \mathrm{d} \boldsymbol{v} \\
& \leq 2 d(d-1) n^{2} \int_{[0,1]^{d} \times[0,1]^{d}} u_{1} u_{2} \exp \left(-n \prod_{i=1}^{d} u_{i}\right) \prod_{i=1}^{d} u_{i} \mathrm{~d} \boldsymbol{u} \mathrm{d} \boldsymbol{v} \\
& =2 d(d-1) n^{2} \int_{[0,1]^{d}} u_{1} u_{2} \exp \left(-n \prod_{i=1}^{d} u_{i}\right) \prod_{i=1}^{d} u_{i} \mathrm{~d} \boldsymbol{u} .
\end{aligned}
$$


Now we make the change of variables $u_{i}=: \mathrm{e}^{-y_{i}}, i=3, \ldots, d$. Then, by the usual argument, we have

$$
\begin{aligned}
J_{2} \leq 2 d(d-1) n^{2} \int_{0}^{1} \mathrm{~d} u_{1} \int_{0}^{1} \mathrm{~d} u_{2} \int_{\left(\mathbb{R}^{+}\right)^{d-3}} u_{1}^{2} u_{2}^{2} \\
\quad \times \exp \left(-n u_{1} u_{2} \exp \left(-\sum_{i=3}^{d} y_{i}\right)\right) \exp \left(-2 \sum_{i=3}^{d} y_{i}\right) \mathrm{d} \boldsymbol{y} \\
=2 d(d-1) n^{2} \int_{0}^{1} \mathrm{~d} u_{1} \int_{0}^{1} \mathrm{~d} u_{2} \int_{0}^{\infty} u_{1}^{2} u_{2}^{2} \exp \left(-n u_{1} u_{2} \mathrm{e}^{-z}\right) \mathrm{e}^{-2 z} \frac{z^{d-3}}{(d-3) !} \mathrm{d} z \\
=O\left((\log n)^{d-3}\right) .
\end{aligned}
$$

For $J_{1}$ we make the following changes of variable: $u_{i}=: \mathrm{e}^{-x_{i}}, v_{i}=: \mathrm{e}^{-y_{i}}, i=2, \ldots, d$, $z:=\sum_{i=2}^{d} x_{i}$, and $w:=\sum_{i=2}^{d} y_{i}$. Then we have

$$
\begin{gathered}
J_{1}=2 d n^{2} \int_{[0,1]^{d} \times[0,1]^{d}} u_{1}^{2} v_{1} \exp \left(-n \prod_{i=1}^{d} u_{i}\left(1+\prod_{i=1}^{d} v_{i}\right)\right) \prod_{i=1}^{d} u_{i} \mathrm{~d} \boldsymbol{u} \mathrm{d} \boldsymbol{v} \\
=2 d n^{2} \int_{0}^{1} \mathrm{~d} u_{1} \int_{0}^{1} \mathrm{~d} v_{1} \iint u_{1}^{3} v_{1} \\
\quad \times \exp \left(-n u_{1} \exp \left(-\sum_{i=2}^{d} x_{i}\right)\left[1+v_{1} \exp \left(-\sum_{i=2}^{d} y_{i}\right)\right]\right) \\
\quad \times \exp \left(-2 \sum_{i=2}^{d} x_{i}-\sum_{i=2}^{d} y_{i}\right) \mathrm{d} \boldsymbol{x} \mathrm{d} \boldsymbol{y} \\
=2 d n^{2} \int_{0}^{1} \mathrm{~d} u_{1} \int_{0}^{1} \mathrm{~d} v_{1} \int_{0}^{\infty} \int_{0}^{\infty} u_{1}^{3} v_{1} \exp \left(-n u_{1} \mathrm{e}^{-z}\left(1+v_{1} \mathrm{e}^{-w}\right)\right) \\
\times \mathrm{e}^{-2 z-w} \frac{z^{d-2}}{(d-2) !} \frac{w^{d-2}}{(d-2) !} \mathrm{d} z \mathrm{~d} w
\end{gathered}
$$

Now, with the changes of variable $z-\log n u_{1}=: a$ and $w-\log v_{1}=: b$, we have

$$
\begin{aligned}
& J_{1}=2 d \int_{0}^{1} \mathrm{~d} u_{1} \int_{0}^{1} \mathrm{~d} v_{1} \int_{-\log n u_{1}}^{\infty} \mathrm{d} a \int_{-\log v_{1}}^{\infty} \mathrm{d} b u_{1} \\
& \times \exp \left(-\mathrm{e}^{-a}\left(1+\mathrm{e}^{-b}\right)\right) \mathrm{e}^{-2 a-b} \frac{\left(a+\log n u_{1}\right)^{d-2}}{(d-2) !} \frac{\left(b+\log v_{1}\right)^{d-2}}{(d-2) !} \\
&=2 d \int_{0}^{1} \mathrm{~d} u_{1} \int_{0}^{1} \mathrm{~d} v_{1} \int_{0}^{n u_{1}} \mathrm{~d} \alpha \int_{0}^{v_{1}} \mathrm{~d} w u_{1} \mathrm{e}^{-\alpha(1+w)} \alpha \\
& \times \frac{\left(-\log \alpha+\log n u_{1}\right)^{d-2}}{(d-2) !} \frac{\left(-\log w+\log v_{1}\right)^{d-2}}{(d-2) !}
\end{aligned}
$$

where $\mathrm{e}^{-a}=: \alpha$ and $\mathrm{e}^{-b}=: w$. We now expand the term

$$
\left(-\log \alpha+\log n u_{1}\right)^{d-2}=\left(-\log \alpha+\log n+\log u_{1}\right)^{d-2}
$$


and integrate term by term. Then, we easily see that integration of the $(\log n)^{d-2}$ term gives the leading term. Thus, as $n \rightarrow \infty$,

$$
\begin{aligned}
J_{1} & \asymp 2 d \int_{0}^{1} \mathrm{~d} u_{1} \int_{0}^{1} \mathrm{~d} v_{1} \int_{0}^{n u_{1}} \mathrm{~d} \alpha \int_{0}^{v_{1}} \mathrm{~d} w u_{1} \mathrm{e}^{-\alpha(1+w)} \alpha \frac{(\log n)^{d-2}}{(d-2) !} \frac{\left(-\log w+\log v_{1}\right)^{d-2}}{(d-2) !} \\
& \asymp 2 d \int_{0}^{1} \mathrm{~d} u_{1} \int_{0}^{1} \mathrm{~d} v_{1} \int_{0}^{\infty} \mathrm{d} \alpha \int_{0}^{v_{1}} \mathrm{~d} w u_{1} \mathrm{e}^{-\alpha(1+w)} \alpha \frac{(\log n)^{d-2}}{(d-2) !} \frac{\left(-\log w+\log v_{1}\right)^{d-2}}{(d-2) !} \\
& =d \int_{0}^{1} \mathrm{~d} v_{1} \int_{0}^{\infty} \mathrm{d} \alpha \int_{0}^{v_{1}} \mathrm{~d} w \mathrm{e}^{-\alpha(1+w)} \alpha \frac{(\log n)^{d-2}}{(d-2) !} \frac{\left(-\log w+\log v_{1}\right)^{d-2}}{(d-2) !} \\
& =d \int_{0}^{1} \mathrm{~d} v_{1} \int_{0}^{v_{1}} \mathrm{~d} w \frac{1}{(1+w)^{2}} \frac{(\log n)^{d-2}}{(d-2) !} \frac{\left(-\log w+\log v_{1}\right)^{d-2}}{(d-2) !} \\
& =\gamma_{d}(\log n)^{d-2},
\end{aligned}
$$

where, recall,

$$
\gamma_{d}=\frac{d}{((d-2) !)^{2}}\left(\int_{0}^{1} \mathrm{~d} v_{1} \int_{0}^{1} \mathrm{~d} s \frac{1}{\left(1+v_{1} s\right)^{2}}\left(\log \frac{1}{s}\right)^{d-2} v_{1}\right) \quad \text { with } w=: v_{1} s .
$$

By (34)-(36) we have

$$
I_{2} \asymp \gamma_{d}(\log n)^{d-2},
$$

and from (17), (18), (31), (33), and (37) we recover Theorem 2.

\section{Central limit theorem}

In this section we prove Theorem 3. With the help of transformation (38), which appeared in [5], we approximate $L_{n}$ by a space-truncated random variable conditioned on a highly probable event $V_{n}$ that we define in (45). Then we approximate this conditioned, space-truncated random variable by a random variable $L_{n}^{\prime \prime}$ generated by a Poisson point process. This Poisson point process approximation idea has been successfully developed in [4]. By decomposing $L_{n}^{\prime \prime}$ as a sum of locally dependent random variables, we can apply Stein's method to $L_{n}^{\prime \prime}$ and obtain the central limit theorem for $L_{n}^{\prime \prime}$. Since our approximation errors turn out to be small, we can extract the central limit theorem for $L_{n}$ (Theorem 3) from the central limit theorem for $L_{n}^{\prime \prime}$.

Let $\boldsymbol{x}_{1}, \ldots, \boldsymbol{x}_{n}$ be independent, identically distributed uniform points in $[0,1]^{d}$. We apply the transformation $g: \boldsymbol{x}=\left(x_{1}, \ldots, x_{d}\right) \mapsto \boldsymbol{y}=\left(y_{1}, \ldots, y_{d}\right)$ such that

$$
y_{i}=-\log x_{i}, \quad i=1, \ldots, d .
$$

Then $\boldsymbol{x}$ is minimal if and only if $\boldsymbol{y}$ is maximal. Furthermore, the distribution of each component of $\boldsymbol{y}$ is exponential with mean 1 .

Define $A_{\zeta}$ and $B_{\zeta}$ as

$$
A_{\zeta}=\left\{\left(y_{1}, \ldots, y_{d}\right) \in \mathbb{R}_{+}^{d}: \sum_{i=1}^{d} y_{i}<\zeta\right\}, \quad B_{\zeta}=\left\{\left(y_{1}, \ldots, y_{d}\right) \in \mathbb{R}_{+}^{d}: \sum_{i=1}^{d} y_{i} \geq \zeta\right\} .
$$

We would like to choose $\alpha_{n}$ and $\beta_{n}, \alpha_{n}<\beta_{n}$, in such a way that there are not many maximal points in $A_{\alpha_{n}}$ and not many points in $B_{\beta_{n}}$. We let

$$
\alpha_{n}=\log n-\log (a \log \log n), \quad \beta_{n}=\log n+b(d-1) \log \log n,
$$


where

$$
a>(d-1)+\frac{1}{2}(d-2), \quad b>1+3 \frac{d-2}{d-1} .
$$

Then we see that

$$
\begin{aligned}
\mathrm{E}\left(\sum_{i=1}^{n} 1\left\{\boldsymbol{y}_{i} \text { maximal and } \boldsymbol{y}_{i} \in A_{\alpha_{n}}\right\}\right) & =n \int_{A_{\alpha_{n}}} \mathrm{e}^{-\left(y_{1}+\cdots+y_{d}\right)}\left(1-\mathrm{e}^{-\left(y_{1}+\cdots+y_{d}\right)}\right)^{n-1} \mathrm{~d} \boldsymbol{y} \\
& \leq n \int_{A_{\alpha_{n}}} \mathrm{e}^{-\left(y_{1}+\cdots+y_{d}\right)} \exp \left(-(n-1) \mathrm{e}^{-\left(y_{1}+\cdots+y_{d}\right)}\right) \mathrm{d} \boldsymbol{y} \\
& =n \int_{0}^{\alpha_{n}} \mathrm{e}^{-s} \exp \left(-(n-1) \mathrm{e}^{-s}\right) \frac{s^{d-1}}{(d-1) !} \mathrm{d} s \\
& \leq n \frac{\alpha_{n}^{d-1}}{(d-1) !} \int_{0}^{\alpha_{n}} \mathrm{e}^{-s} \exp \left(-n \mathrm{e}^{-s}\right) \mathrm{d} s \\
& =n \frac{\alpha_{n}^{d-1}}{(d-1) !} \int_{\mathrm{e}^{-\alpha_{n}}}^{1} \mathrm{e}^{-n t} \mathrm{~d} t \\
& \leq \frac{\alpha_{n}^{d-1}}{(d-1) !} \exp \left(-n \mathrm{e}^{-\alpha_{n}}\right) \\
& =O((\log n)
\end{aligned}
$$

and

$$
\begin{aligned}
\mathrm{E}\left(\sum_{i=1}^{n} 1\left\{\boldsymbol{y}_{i} \in B_{\beta_{n}}\right\}\right) & =n \int_{B_{\beta_{n}}} \mathrm{e}^{-\left(y_{1}+\cdots+y_{d}\right)} \mathrm{d} \boldsymbol{y} \\
& =n \int_{\beta_{n}}^{\infty} \mathrm{e}^{-s} \frac{s^{d-1}}{(d-1) !} \mathrm{d} s \\
& \asymp n \mathrm{e}^{-\beta_{n}} \frac{\beta_{n}^{d-1}}{(d-1) !} \\
& =O\left((\log n)^{-(b-1)(d-1)}\right) \quad(\text { by (39) and (40)). }
\end{aligned}
$$

Define

$$
\tilde{L}_{n}:=\sum_{i=1}^{n}\left|\boldsymbol{x}_{i}\right| 1_{G_{i}} 1\left\{\boldsymbol{y}_{i} \in B_{\alpha_{n}} \cap A_{\beta_{n}}\right\}
$$

and define $L_{n}^{\prime}$ as a conditional distribution of $\tilde{L}_{n}$ given $V_{n}$. In other words, with density function $f_{n}(\boldsymbol{x})$ for $\tilde{L}_{n}$, let

$$
L_{n}^{\prime}:= \begin{cases}\tilde{L}_{n} \text { with density function } f_{n}(\boldsymbol{x}) / \mathrm{P}\left(V_{n}\right) & \text { if } V_{n} \text { occurs } \\ \tilde{L}_{n} \text { with density function } 0 & \text { if } V_{n} \text { does not occur }\end{cases}
$$

where

$$
V_{n}:=\bigcap_{i=1}^{n}\left\{x_{i} \in A_{\beta_{n}}\right\}
$$

With these definitions, we have

$$
\mathrm{P}\left(L_{n}^{\prime} \in A\right)=\mathrm{P}\left(\tilde{L}_{n} \in A \mid V_{n}\right) .
$$


By the estimates given in (42) and (43), and employing the $\delta$-method (see [9]), the convergence rate of the distribution of $L_{n}$ should be the same as that of the distribution of $L_{n}^{\prime}$. Furthermore, the distribution of $L_{n}^{\prime}$ should be asymptotically equivalent to the distribution of

$$
L_{n}^{\prime \prime}:=\sum_{\boldsymbol{y} \in W_{n}}\left|g^{-1}(\boldsymbol{y})\right| 1\left\{\boldsymbol{y} \text { is maximal in } W_{n}\right\},
$$

where $W_{n}$ is a Poisson process on $B_{\alpha_{n}} \cap A_{\beta_{n}}$ with intensity $n \mathrm{e}^{-\left(y_{1}+\cdots+y_{d}\right)} / \mathrm{P}\left(V_{n}\right)$. Therefore, our plan is to first prove the central limit theorem for $L_{n}^{\prime \prime}$ using Stein's method. From this central limit theorem we shall obtain the central limit theorem for $L_{n}^{\prime}$ and then $L_{n}$.

We rewrite $L_{n}$ as

$$
L_{n}=K_{n, 1}+K_{n, 2}=J_{n, 1}+J_{n, 2}+K_{n, 2},
$$

where

$$
\begin{array}{cl}
K_{n, 1}=\sum_{i=1}^{n}\left|\boldsymbol{x}_{i}\right| 1_{G_{i}} 1\left\{\boldsymbol{y}_{i} \in B_{\alpha_{n}}\right\}, & K_{n, 2}=\sum_{i=1}^{n}\left|\boldsymbol{x}_{i}\right| 1_{G_{i}} 1\left\{\boldsymbol{y}_{i} \in A_{\alpha_{n}}\right\}, \\
J_{n, 1}=K_{n, 1} 1\left(V_{n}\right), & J_{n, 2}=K_{n, 1} 1\left(V_{n}^{\mathrm{c}}\right) .
\end{array}
$$

Then, since by (42), (43), and (40) we have

$$
\begin{gathered}
\mathrm{P}\left(K_{n, 2} \neq 0\right) \leq \mathrm{E}\left(\sum_{i=1}^{n} 1_{G_{i}} 1\left\{\boldsymbol{y}_{i} \in A_{\alpha_{n}}\right\}\right) \leq O\left((\log n)^{-(a-(d-1))}\right), \\
\mathrm{P}\left(V_{n}^{\mathrm{c}}\right) \leq \mathrm{E}\left(\sum_{i=1}^{n} 1\left\{\boldsymbol{y}_{i} \in B_{\beta_{n}}\right\}\right)=O\left((\log n)^{-(b-1)(d-1)}\right),
\end{gathered}
$$

by (46) we have the following upper bound for the total variation distance, $d_{\mathrm{TV}}\left(L_{n}, L_{n}^{\prime}\right)$, between $L_{n}$ and $L_{n}^{\prime}$ :

$$
\begin{aligned}
d_{\mathrm{TV}}\left(L_{n}, L_{n}^{\prime}\right) & =\sup _{A}\left|\mathrm{P}\left(L_{n} \in A\right)-\mathrm{P}\left(L_{n}^{\prime} \in A\right)\right| \\
& =\sup _{A}\left|\mathrm{P}\left(L_{n} \in A\right)-\mathrm{P}\left(\tilde{L}_{n} \in A \mid V_{n}\right)\right| \\
& =\sup _{A}\left|\mathrm{P}\left(L_{n} \in A\right)-\frac{\mathrm{P}\left(\tilde{L}_{n} \in A, V_{n}\right)}{\mathrm{P}\left(V_{n}\right)}\right| \\
& =\sup _{A}\left|\frac{\mathrm{P}\left(V_{n}\right) \mathrm{P}\left(L_{n} \in A\right)-\mathrm{P}\left(\tilde{L}_{n} \in A, V_{n}\right)}{\mathrm{P}\left(V_{n}\right)}\right| \\
& =\sup _{A}\left|\frac{\mathrm{P}\left(V_{n}\right)\left(\mathrm{P}\left(L_{n} \in A, V_{n}\right)+\mathrm{P}\left(L_{n} \in A, V_{n}^{\mathrm{c}}\right)\right)-\left(\mathrm{P}\left(V_{n}\right)+\mathrm{P}\left(V_{n}^{\mathrm{c}}\right)\right) \mathrm{P}\left(\tilde{L}_{n} \in A, V_{n}\right)}{\mathrm{P}\left(V_{n}\right)}\right| \\
& \leq \sup _{A}\left|\mathrm{P}\left(L_{n} \in A, V_{n}\right)-\mathrm{P}\left(\tilde{L}_{n} \in A, V_{n}\right)\right|+\sup _{A} \mathrm{P}\left(L_{n} \in A, V_{n}^{\mathrm{c}}\right)+\frac{\mathrm{P}\left(V_{n}^{\mathrm{c}}\right)}{\mathrm{P}\left(V_{n}\right)} \\
& \leq \sup _{A}\left|\mathrm{P}\left(L_{n} \in A, V_{n}\right)-\mathrm{P}\left(\tilde{L}_{n} \in A, V_{n}\right)\right|+\mathrm{P}\left(V_{n}^{\mathrm{c}}\right)+\frac{\mathrm{P}\left(V_{n}^{\mathrm{c}}\right)}{\mathrm{P}\left(V_{n}\right)} \\
& \leq \mathrm{P}\left(K_{n, 2} \neq 0\right)+2 \frac{\mathrm{P}\left(V_{n}^{\mathrm{c}}\right)}{\mathrm{P}\left(V_{n}\right)} \\
\leq & O\left((\log n)^{-(a-(d-1))}+(\log n)^{-(b-1)(d-1)}\right) .
\end{aligned}
$$


Moreover, with the notation $p_{n}:=\mathrm{P}\left(V_{n}\right)$, we have

$$
\begin{aligned}
1-p_{n} & =\mathrm{P}\left(V_{n}^{\mathrm{c}}\right) \leq C(\log n)^{-(b-1)(d-1)}, \\
\frac{1}{p_{n}}-1 & \leq C(\log n)^{-(b-1)(d-1)}, \\
\frac{1}{p_{n}}+1 & \leq 2+C(\log n)^{-(b-1)(d-1)} \leq C .
\end{aligned}
$$

Next, we claim that

$$
\begin{aligned}
\mathrm{E}\left(J_{n, 2}\right) & \leq C(\log n)^{(d-2)-(b-1)(d-1)}, \\
\mathrm{E}\left(K_{n, 2}\right) & \leq C(\log n)^{-(a-(d-1))} \\
\mathrm{E}\left(J_{n, 2}^{2}\right) & \leq C(\log n)^{-(b-1)(d-1)+2(d-2)}, \\
\mathrm{E}\left(K_{n, 2}^{2}\right) & \leq C(\log n)^{-(a-(d-1))}
\end{aligned}
$$

Let

$$
F_{i}:=\left\{\boldsymbol{y}_{i} \in B_{\beta_{n}}\right\}
$$

Since $L_{n-1}$ and $F_{n}$ are independent and since, by (43),

$$
\mathrm{P}\left(F_{n}\right) \leq C n^{-1}(\log n)^{-(b-1)(d-1)},
$$

by Theorem 1 we have (51):

$$
\begin{aligned}
\mathrm{E}\left(J_{n, 2}\right) & \leq \mathrm{E}\left(L_{n}\left(\sum_{i=1}^{n} 1_{F_{i}}\right)\right) \\
& =n \mathrm{E}\left(L_{n} 1_{F_{n}}\right) \\
& \leq n \mathrm{E}\left(\left(L_{n-1}+d^{1 / 2}\right) 1_{F_{n}}\right) \\
& =n \mathrm{E}\left(L_{n-1}+d^{1 / 2}\right) \mathrm{P}\left(F_{n}\right) \\
& \leq C(\log n)^{-(b-1)(d-1)+(d-2)} .
\end{aligned}
$$

By the same argument, using Theorems 1 and 2 yields (53):

$$
\begin{aligned}
\mathrm{E}\left(J_{n, 2}^{2}\right) & \leq \mathrm{E}\left(L_{n}^{2}\left(\sum_{i=1}^{n} 1_{F_{i}}\right)\right) \\
& =n \mathrm{E}\left(L_{n}^{2} 1_{F_{n}}\right) \\
& \leq n \mathrm{E}\left(\left(L_{n-1}+d^{1 / 2}\right)^{2} 1_{F_{n}}\right) \\
& \leq n \mathrm{E}\left(\left(L_{n-1}+d^{1 / 2}\right)^{2}\right) \mathrm{P}\left(F_{n}\right) \\
& =n\left(\mathrm{E}\left(L_{n-1}^{2}\right)+2 d^{1 / 2} \mathrm{E}\left(L_{n-1}\right)+d\right) \mathrm{P}\left(F_{n}\right) \\
& \leq C(\log n)^{-(b-1)(d-1)+2(d-2)} .
\end{aligned}
$$


Furthermore, (52) follows from (42). To prove (54), we start with a simple observation. Let $Q_{\boldsymbol{y}}$ be the first orthant of $\boldsymbol{y}$, that is $Q_{\boldsymbol{y}}=\{\boldsymbol{z}: \boldsymbol{z} \succ \boldsymbol{y}\}$. Then, with the notation $\|\boldsymbol{y}\|=y_{1}+\cdots+y_{d}$, the probability that $\boldsymbol{y}_{1}$ lies in $Q_{\boldsymbol{y}}$ is given by

$$
\mathrm{P}\left(\boldsymbol{y}_{1} \in Q_{\boldsymbol{y}}\right)=\prod_{j=1}^{d} \mathrm{e}^{-y_{j}}=\mathrm{e}^{-\|\boldsymbol{y}\|} .
$$

Now, using the fact that $\mathrm{P}(A \cup B) \geq(\mathrm{P}(A)+\mathrm{P}(B)) / 2$ for any two events $A$ and $B$, we find that, given $\boldsymbol{y}_{1}$ and $\boldsymbol{y}_{2}$, the conditional probability that both $\boldsymbol{y}_{1}$ and $\boldsymbol{y}_{2}$ are maximal is bounded by

$$
\left(1-\frac{1}{2}\left(\mathrm{e}^{-\left\|\boldsymbol{y}_{1}\right\|}+\mathrm{e}^{-\left\|\boldsymbol{y}_{2}\right\|}\right)\right)^{n-2} \leq \exp \left(-(n-2) \frac{1}{2}\left(\mathrm{e}^{-\left\|\boldsymbol{y}_{1}\right\|}+\mathrm{e}^{-\left\|\boldsymbol{y}_{2}\right\|}\right)\right) .
$$

Thus, by a computation similar to that of (41), we have (54):

$$
\begin{aligned}
\mathrm{E}\left(K_{n, 2}^{2}\right) & =\mathrm{E}\left(\left(\sum_{i=1}^{n}\left|\boldsymbol{x}_{i}\right| 1\left\{\boldsymbol{y}_{i} \text { is maximal and }\left\|\boldsymbol{y}_{i}\right\| \leq \alpha_{n}\right\}\right)^{2}\right) \\
& \leq C \mathrm{E}\left(K_{n, 2}\right)+C n^{2} \mathrm{P}\left(\text { both } \boldsymbol{y}_{1} \text { and } \boldsymbol{y}_{2} \text { are maximal and lie in } A_{\alpha_{n}}\right) \\
& \leq C \mathrm{E}\left(K_{n, 2}\right)+C n^{2}\left(\frac{1}{(d-1) !}\right)^{2} \int_{0}^{\alpha_{n}} \int_{0}^{\alpha_{n}}(x y)^{d-1} \exp \left(-(n-2) \frac{\mathrm{e}^{-x}+\mathrm{e}^{-y}}{2}\right) \\
& =C \mathrm{E}\left(K_{n, 2}\right)+C n^{2}\left(\frac{1}{(d-1) !} \int_{0}^{\alpha_{n}} x^{d-1} \exp \left(-(n-2) \frac{\mathrm{e}^{-x}}{2}\right) \mathrm{e}^{-x} \mathrm{~d} x\right)^{2} \\
& \leq C \mathrm{E}\left(K_{n, 2}\right)+C n^{2}\left(\frac{1}{(d-1) !} \alpha_{n}^{d-1} \int_{0}^{\alpha_{n}} \exp \left(-(n-2) \frac{\mathrm{e}^{-x}}{2}\right) \mathrm{e}^{-x} \mathrm{~d} x\right)^{2} \\
& =C \mathrm{E}\left(K_{n, 2}\right)+C n^{2}\left(\frac{1}{(d-1) !} \alpha_{n}^{d-1} \int_{\mathrm{e}^{-\alpha_{n}}}^{1} \mathrm{e}^{-(n-2) t / 2} \mathrm{~d} t\right)^{2} \\
& \leq C(\log n)^{-(a-(d-1))}+C(\log n)^{-2([(n-2) / n] a-(d-1))} \\
& \leq C(\log n)^{-(a-(d-1))} .
\end{aligned}
$$

Now, since by (44), (45), and (47) we have $\mathrm{E}\left(L_{n}^{\prime}\right)=p_{n}^{-1} \mathrm{E}\left(J_{n, 1}\right)$ and $\mathrm{E}\left(L_{n}^{\prime 2}\right)=p_{n}^{-1} \mathrm{E}\left(J_{n, 1}^{2}\right)$, by (49), Theorem 1, (51), and (52) we have

$$
\begin{aligned}
\left|\mathrm{E}\left(L_{n}\right)-\mathrm{E}\left(L_{n}^{\prime}\right)\right| & \leq\left(\frac{1}{p_{n}}-1\right) \mathrm{E}\left(J_{n, 1}\right)+\mathrm{E}\left(J_{n, 2}\right)+\mathrm{E}\left(K_{n, 2}\right) \\
& \leq\left(\frac{1}{p_{n}}-1\right) \mathrm{E}\left(L_{n}\right)+\mathrm{E}\left(J_{n, 2}\right)+\mathrm{E}\left(K_{n, 2}\right) \\
& \leq C(\log n)^{(d-2)-(b-1)(d-1)}+C(\log n)^{-(a-(d-1))} .
\end{aligned}
$$

By (50) and Theorem 1 we have

$$
\mathrm{E}\left(L_{n}\right)+\mathrm{E}\left(L_{n}^{\prime}\right)=\mathrm{E}\left(L_{n}\right)+\frac{1}{p_{n}} \mathrm{E}\left(J_{n, 1}\right) \leq\left(1+\frac{1}{p_{n}}\right) \mathrm{E}\left(L_{n}\right) \leq C(\log n)^{(d-2)},
$$


by (49), Theorems 1 and 2, (53), and (54) we have

$$
\begin{aligned}
\left|\mathrm{E}\left(L_{n}^{2}\right)-\mathrm{E}\left(L_{n}^{\prime 2}\right)\right| & \leq\left(\frac{1}{p_{n}}-1\right) \mathrm{E}\left(J_{n, 1}^{2}\right)+\mathrm{E}\left(J_{n, 2}^{2}\right)+\mathrm{E}\left(K_{n, 2}^{2}\right)+2 \mathrm{E}\left(K_{n, 1} K_{n, 2}\right) \\
& \leq\left(\frac{1}{p_{n}}-1\right) \mathrm{E}\left(J_{n, 1}^{2}\right)+\mathrm{E}\left(J_{n, 2}^{2}\right)+\mathrm{E}\left(K_{n, 2}^{2}\right)+2\left(\mathrm{E}\left(K_{n, 1}^{2}\right)\right)^{1 / 2}\left(\mathrm{E}\left(K_{n, 2}^{2}\right)\right)^{1 / 2} \\
& \leq\left(\frac{1}{p_{n}}-1\right) \mathrm{E}\left(L_{n}^{2}\right)+\mathrm{E}\left(J_{n, 2}^{2}\right)+\mathrm{E}\left(K_{n, 2}^{2}\right)+2\left(\mathrm{E}\left(L_{n}^{2}\right)\right)^{1 / 2}\left(\mathrm{E}\left(K_{n, 2}^{2}\right)\right)^{1 / 2} \\
& \leq C(\log n)^{2(d-2)-(b-1)(d-1)}+C(\log n)^{(d-2)-(a-(d-1)) / 2}
\end{aligned}
$$

and by (55)-(57) we have

$$
\begin{aligned}
\left|\operatorname{var}\left(L_{n}\right)-\operatorname{var}\left(L_{n}^{\prime}\right)\right| & \leq\left|\mathrm{E}\left(L_{n}^{2}\right)-\mathrm{E}\left(L_{n}^{\prime 2}\right)\right|+\left|\left(\mathrm{E}\left(L_{n}\right)\right)^{2}-\left(\mathrm{E}\left(L_{n}^{\prime}\right)\right)^{2}\right| \\
& =\left|\mathrm{E}\left(L_{n}^{2}\right)-\mathrm{E}\left(L_{n}^{\prime 2}\right)\right|+\left|\mathrm{E}\left(L_{n}\right)-\mathrm{E}\left(L_{n}^{\prime}\right)\right|\left|\mathrm{E}\left(L_{n}\right)+\mathrm{E}\left(L_{n}^{\prime}\right)\right| \\
& \leq C(\log n)^{2(d-2)-(b-1)(d-1)}+C(\log n)^{(d-2)-(a-(d-1)) / 2} .
\end{aligned}
$$

Now define

$$
\tilde{N}_{n}:=\left|\left\{\boldsymbol{y}_{i}, 1 \leq i \leq n\right\} \cap\left(B_{\alpha_{n}} \cap A_{\beta_{n}}\right)\right|
$$

and define $N_{n}^{\prime}$ as a conditional distribution of $\tilde{N}_{n}$ given $V_{n}$. In other words, with mass function $e_{n}(\boldsymbol{x})$ for $\tilde{N}_{n}$, let

$$
N_{n}^{\prime}:= \begin{cases}\tilde{N}_{n} \text { with mass function } e_{n}(\boldsymbol{x}) / \mathrm{P}\left(V_{n}\right) & \text { if } V_{n} \text { occurs } \\ \tilde{N}_{n} \text { with mass function } 0 & \text { if } V_{n} \text { does not occur. }\end{cases}
$$

Then, since $\mathrm{P}\left(L_{n}^{\prime} \in A \mid N_{n}^{\prime}=m\right)=\mathrm{P}\left(L_{n}^{\prime \prime} \in A \mid N_{n}^{\prime \prime}=m\right)$, we have

$$
\begin{aligned}
& d_{\mathrm{TV}}\left(L_{n}^{\prime}, L_{n}^{\prime \prime}\right) \\
& \quad=\sup _{A}\left|\mathrm{P}\left(L_{n}^{\prime} \in A\right)-\mathrm{P}\left(L_{n}^{\prime \prime} \in A\right)\right| \\
& \quad=\sup _{A}\left|\sum_{m=0}^{n} \mathrm{P}\left(N_{n}^{\prime}=m\right) \mathrm{P}\left(L_{n}^{\prime} \in A \mid N_{n}^{\prime}=m\right)-\sum_{m=0}^{\infty} \mathrm{P}\left(N_{n}^{\prime \prime}=m\right) \mathrm{P}\left(L_{n}^{\prime \prime} \in A \mid N_{n}^{\prime \prime}=m\right)\right| \\
& \quad \leq \sum_{m=0}^{n}\left|\mathrm{P}\left(N_{n}^{\prime}=m\right)-\mathrm{P}\left(N_{n}^{\prime \prime}=m\right)\right|+\mathrm{P}\left(N_{n}^{\prime \prime}>n\right) \\
& =2 d_{\mathrm{TV}}\left(X_{n}, Y_{n}\right)
\end{aligned}
$$

where $X_{n}$ is the binomial distribution with $n$ trials and success rate

$$
q_{n}=\frac{\int_{\alpha_{n}}^{\beta_{n}}\left[x^{d-1} /(d-1) !\right] \mathrm{e}^{-x} \mathrm{~d} x}{\int_{0}^{\beta_{n}}\left[x^{d-1} /(d-1) !\right] \mathrm{e}^{-x} \mathrm{~d} x}=O\left(n^{-1}(\log n)^{d-1} \log \log n\right)
$$

and $Y_{n}$ is the Poisson distribution with mean $\lambda=n q_{n}$. Since $d_{\mathrm{TV}}\left(X_{n}, Y_{n}\right) \leq(\lambda \vee 1)^{-1} n q_{n}^{2}$ and since $\lambda=n q_{n}$ is large for large $n$, we have

$$
d_{\mathrm{TV}}\left(L_{n}^{\prime}, L_{n}^{\prime \prime}\right) \leq 2 d_{\mathrm{TV}}\left(X_{n}, Y_{n}\right) \leq 2 q_{n}=O\left(n^{-1}(\log n)^{d-1} \log \log n\right) .
$$


Similarly, since $\mathrm{E}\left(L_{n}^{\prime} \mid N_{n}^{\prime}=m\right)=\mathrm{E}\left(L_{n}^{\prime \prime} \mid N_{n}^{\prime \prime}=m\right), \mathrm{E}\left(L_{n}^{\prime \prime} \mid N_{n}^{\prime \prime}=m\right) \leq C m$ and, for large $n$,

$$
\sum_{m=0}^{n}\left|\mathrm{P}\left(N_{n}^{\prime}=m\right)-\mathrm{P}\left(N_{n}^{\prime \prime}=m\right)\right|+\mathrm{P}\left(N_{n}^{\prime \prime}>n\right)=2 d_{\mathrm{TV}}\left(X_{n}, Y_{n}\right) \leq 2 q_{n},
$$

we find, for large $n$, that

$$
\begin{aligned}
& \left|\mathrm{E}\left(L_{n}^{\prime}\right)-\mathrm{E}\left(L_{n}^{\prime \prime}\right)\right|=\left|\sum_{m=0}^{n} \mathrm{P}\left(N_{n}^{\prime}=m\right) \mathrm{E}\left(L_{n}^{\prime} \mid N_{n}^{\prime}=m\right)-\sum_{m=0}^{\infty} \mathrm{P}\left(N_{n}^{\prime \prime}=m\right) \mathrm{E}\left(L_{n}^{\prime \prime} \mid N_{n}^{\prime \prime}=m\right)\right| \\
& \leq \sum_{m=0}^{\left\lfloor 2 n q_{n}\right\rfloor}\left|\mathrm{P}\left(N_{n}^{\prime}=m\right)-\mathrm{P}\left(N_{n}^{\prime \prime}=m\right)\right| E\left(L_{n}^{\prime \prime} \mid N_{n}^{\prime \prime}=m\right) \\
& +\sum_{m=\left\lfloor 2 n q_{n}\right\rfloor+1}^{n} \mathrm{P}\left(N_{n}^{\prime}=m\right) \mathrm{E}\left(L_{n}^{\prime} \mid N_{n}^{\prime}=m\right) \\
& +\sum_{m=\left\lfloor 2 n q_{n}\right\rfloor+1}^{\infty} \mathrm{P}\left(N_{n}^{\prime \prime}=m\right) \mathrm{E}\left(L_{n}^{\prime \prime} \mid N_{n}^{\prime \prime}=m\right) \\
& \leq C \sum_{m=0}^{\left\lfloor 2 n q_{n}\right\rfloor}\left|\mathrm{P}\left(N_{n}^{\prime}=m\right)-\mathrm{P}\left(N_{n}^{\prime \prime}=m\right)\right| 2 n q_{n} \\
& +C \sum_{m=\left\lfloor 2 n q_{n}\right\rfloor+1}^{n} \mathrm{P}\left(N_{n}^{\prime}=m\right) m \\
& +C \sum_{m=\left\lfloor 2 n q_{n}\right\rfloor+1}^{\infty} \mathrm{P}\left(N_{n}^{\prime \prime}=m\right) m \\
& \leq C \sum_{m=0}^{\infty}\left|\mathrm{P}\left(N_{n}^{\prime}=m\right)-\mathrm{P}\left(N_{n}^{\prime \prime}=m\right)\right| 2 n q_{n} \\
& +C \sum_{m=\left\lfloor 2 n q_{n}\right\rfloor+1}^{n} \mathrm{P}\left(N_{n}^{\prime}=m\right) m+C \sum_{m=\left\lfloor 2 n q_{n}\right\rfloor+1}^{\infty} \mathrm{P}\left(N_{n}^{\prime \prime}=m\right) m \\
& \leq C n q_{n}^{2}+C \sum_{m=\left\lfloor 2 n q_{n}\right\rfloor+1}^{n} \mathrm{P}\left(N_{n}^{\prime}=m\right) m+C \sum_{m=\left\lfloor 2 n q_{n}\right\rfloor+1}^{\infty} \mathrm{P}\left(N_{n}^{\prime \prime}=m\right) m \text {, }
\end{aligned}
$$

where $\lfloor\cdot\rfloor$ is the greatest-integer function. Since, by the tail estimate of the binomial distribution,

$$
\begin{aligned}
\sum_{m=\left\lfloor 2 n q_{n}\right\rfloor+1}^{n} \mathrm{P}\left(N_{n}^{\prime}=m\right) m & =\mathrm{E}\left(X_{n}, X_{n}>2 n q_{n}\right) \\
& =\mathrm{E}\left(X_{n}-\mathrm{E}\left(X_{n}\right), X_{n}>2 n q_{n}\right)+n q_{n} \mathrm{P}\left(X_{n}>2 n q_{n}\right) \\
& \leq\left(n q_{n}\left(1-q_{n}\right)\right)^{1 / 2}\left(\mathrm{P}\left(X_{n}>2 n q_{n}\right)\right)^{1 / 2}+n q_{n} \mathrm{P}\left(X_{n}>2 n q_{n}\right) \\
& =O\left(\left(n q_{n}\right)^{1 / 4} \mathrm{e}^{-n q_{n} / 4}\right),
\end{aligned}
$$


and since, by the tail estimate of the Poisson distribution (by Stirling's formula),

$$
\begin{aligned}
\sum_{m=\left\lfloor 2 n q_{n}\right\rfloor+1}^{\infty} \mathrm{P}\left(N_{n}^{\prime \prime}=m\right) m & =\lambda \sum_{m=\left\lfloor 2 n q_{n}\right\rfloor}^{\infty} \mathrm{e}^{-\lambda} \frac{\lambda^{m}}{m !} \\
& \leq C \lambda \mathrm{e}^{-\lambda} \frac{\lambda^{\left\lfloor 2 n q_{n}\right\rfloor}}{\left\lfloor 2 n q_{n}\right\rfloor !} \\
& =O\left(\left(n q_{n}\right)^{1 / 2}(\mathrm{e} / 4)^{n q_{n}}\right),
\end{aligned}
$$

by (59) we have

$$
\left|\mathrm{E}\left(L_{n}^{\prime}\right)-\mathrm{E}\left(L_{n}^{\prime \prime}\right)\right| \leq C n q_{n}^{2} \leq C n^{-1}(\log n)^{2(d-1)}(\log \log n)^{2} .
$$

By a similar calculation, we also have

$$
\left|\mathrm{E}\left(L_{n}^{\prime}\left(L_{n}^{\prime}-1\right)\right)-\mathrm{E}\left(L_{n}^{\prime \prime}\left(L_{n}^{\prime \prime}-1\right)\right)\right| \leq C n^{2} q_{n}^{3} \leq C n^{-1}(\log n)^{3(d-1)}(\log \log n)^{3} .
$$

Next, we split $\mathbb{R}_{+}^{d}$ into cubes, $T_{i}$, of edge length $l \equiv l(n)$, where we choose $l$ to be sufficiently small that the argument in (92), below, makes sense. At this point, simply think of $l$ as a fixed but small number, even though the choice of $l$ depends on $n$. Let $Z_{i}$ be the contribution to $L_{n}^{\prime \prime}$ of the Poisson point process lying in the cell $T_{i}$ :

$$
Z_{i}:=\sum_{\boldsymbol{y} \in\left(W_{n} \cap T_{i}\right)}\left|g^{-1}(\boldsymbol{y})\right| 1\left\{\boldsymbol{y} \text { is maximal in } W_{n}\right\} .
$$

Then we can rewrite $L_{n}^{\prime \prime}$ as

$$
L_{n}^{\prime \prime}=\sum_{T_{i} \cap\left(B_{\alpha_{n}} \cap A_{\beta_{n}}\right) \neq \varnothing} Z_{i} .
$$

Since we have decomposed $L_{n}^{\prime \prime}$ into a sum of locally dependent random variables, we apply Stein's method to $L_{n}^{\prime \prime}$ to obtain the central limit theorem for $L_{n}^{\prime \prime}$. Here is the simple version of Stein's method that we use (Theorem 6.31 of [11]).

Lemma 1. Let $X_{i}$ be a collection of locally dependent random variables with $\mathrm{E}\left(X_{i}^{2}\right)<\infty$, and let

$$
\begin{array}{cc}
U_{i}=\left\{j: X_{j} \text { depends on } X_{i}\right\}, & V_{i}=\sum_{j \in U_{i}} X_{j}, \\
U_{i, j}=\left\{k: X_{k} \text { depends on } X_{i} \text { or } X_{j}\right\} \backslash U_{i}, \quad V_{i, j}=\sum_{k \in U_{i, j}} X_{k}, \quad j \in U_{i}, \\
S=\sum_{i} X_{i}, \quad S_{i}=S-V_{i}, \quad S_{i, j}=S-V_{i, j} .
\end{array}
$$

Suppose that

$$
\mathrm{E}\left(X_{i}\right)=0 \text { for all } i
$$

and

$$
\mathrm{E}\left(S^{2}\right)=\sum_{i} \mathrm{E}\left(X_{i} V_{i}\right)=\sum_{i} \sum_{j \in U_{i}} \mathrm{E}\left(X_{i} X_{j}\right)=1 .
$$


Then, for any function $h$ with $\sup _{x}|h(x)|+\sup _{x}\left|h^{\prime}(x)\right| \leq 1$, we have

$$
|\mathrm{E}(h(S))-\mathrm{E}(h(N))| \leq C \sum_{i} \sum_{j \in U_{i}} \sum_{k \in U_{i} \cup U_{i, j}}\left(\mathrm{E}\left(\left|X_{i} X_{j} X_{k}\right|\right)+\mathrm{E}\left(\left|X_{i} X_{j}\right|\right) \mathrm{E}\left(\left|X_{k}\right|\right)\right),
$$

where $N$ is the standard normal random variable.

Proposition 1. The normalized random variable $\left(L_{n}^{\prime \prime}-\mathrm{E}\left(L_{n}^{\prime \prime}\right)\right) /\left(\operatorname{var}\left(L_{n}^{\prime \prime}\right)\right)^{1 / 2}$ converges in distribution to the standard normal with rate

$$
d_{1}\left(\frac{L_{n}^{\prime \prime}-\mathrm{E}\left(L_{n}^{\prime \prime}\right)}{\left(\operatorname{var}\left(L_{n}^{\prime \prime}\right)\right)^{1 / 2}}, N(0,1)\right)=O\left((\log n)^{-(d-2) / 2}(\log \log n)^{d+1}\right),
$$

where

$$
d_{1}(X, Y):=\sup \left\{|\mathrm{E}(h(X))-\mathrm{E}(h(Y))|: \sup _{x}|h(x)|+\sup _{x}\left|h^{\prime}(x)\right| \leq 1\right\} .
$$

Proof. Let $X_{i}=\left(Z_{i}-\mathrm{E}\left(Z_{i}\right)\right) /\left(\operatorname{var}\left(L_{n}^{\prime \prime}\right)\right)^{1 / 2}$. Then the $X_{i}$ satisfy (63) and (64). Thus, by Lemma 1 , for any function $h$ with $\sup _{x}|h(x)|+\sup _{x}\left|h^{\prime}(x)\right| \leq 1$, we have

$$
\begin{aligned}
& \left|\mathrm{E}\left(h\left(\frac{L_{n}^{\prime \prime}-\mathrm{E}\left(L_{n}^{\prime \prime}\right)}{\left(\operatorname{var}\left(L_{n}^{\prime \prime}\right)\right)^{1 / 2}}\right)\right)-\mathrm{E}(h(N))\right| \\
& \quad \leq C\left(\operatorname{var}\left(L_{n}^{\prime \prime}\right)\right)^{-3 / 2} \sum_{i} \sum_{j \in U_{i}} \sum_{k \in U_{i} \cup U_{i, j}}\left(\mathrm{E}\left(Z_{i} Z_{j} Z_{k}\right)+\mathrm{E}\left(Z_{i}\right) \mathrm{E}\left(Z_{j} Z_{k}\right)+\mathrm{E}\left(Z_{j}\right) \mathrm{E}\left(Z_{i} Z_{k}\right)\right. \\
& \left.+\mathrm{E}\left(Z_{k}\right) \mathrm{E}\left(Z_{i} Z_{j}\right)+\mathrm{E}\left(Z_{i}\right) \mathrm{E}\left(Z_{j}\right) \mathrm{E}\left(Z_{k}\right)\right) .
\end{aligned}
$$

Now define the constants

$$
\begin{gathered}
Q_{n}=\max _{i, j \in U_{i}} \sum_{k \in U_{i} \cup U_{i, j}} \mathrm{E}\left(N_{k}\right), \\
\varepsilon_{n, 1}=\max _{i}\left(r_{i}\right), \quad \varepsilon_{n, 2}=\max _{i}\left(r_{i}\right) \sum_{i} r_{i}=\varepsilon_{n, 1} n q_{n},
\end{gathered}
$$

where $N_{i}$ is the number of Poisson points lying in the region $T_{i}$ and $r_{i}=\mathrm{E}\left(N_{i}\right)$.

We now consider the term $\mathrm{E}\left(Z_{i} Z_{j} Z_{k}\right)$ in (66). If $i, j$, and $k$ are distinct then

$$
\mathrm{E}\left(Z_{i} Z_{j} Z_{k}\right) \leq C \mathrm{E}\left(Z_{i}\right) \mathrm{E}\left(N_{j}\right) \mathrm{E}\left(N_{k}\right) .
$$

It is obvious that $\mathrm{E}\left(Z_{i} Z_{j} \mid N_{k}=m\right)$ is a decreasing function of $m$. Thus, $\mathrm{E}\left(Z_{i} Z_{j} \mid N_{k}\right)$ and $N_{k}$ are negatively correlated and, since $Z_{k} \leq C N_{k}$, we have

$$
\mathrm{E}\left(Z_{i} Z_{j} Z_{k}\right) \leq C \mathrm{E}\left(Z_{i} Z_{j} N_{k}\right)=C \mathrm{E}\left(\mathrm{E}\left(Z_{i} Z_{j} \mid N_{k}\right) N_{k}\right) \leq C \mathrm{E}\left(Z_{i} Z_{j}\right) \mathrm{E}\left(N_{k}\right) .
$$

By the same reasoning, we have $\mathrm{E}\left(Z_{i} Z_{j}\right) \leq C \mathrm{E}\left(Z_{i}\right) \mathrm{E}\left(N_{j}\right)$. Hence, (67) indeed holds. If two of the three indices $i, j$, and $k$ are equal, then there are three cases to consider. In the first case, $\mathrm{E}\left(Z_{i} Z_{j}^{2}\right)$ is bounded (according to $\left.(67)\right)$ by

$$
\begin{aligned}
\mathrm{E}\left(Z_{i} Z_{j}^{2}\right) & \leq C \mathrm{E}\left(Z_{i} N_{j}^{2}\right) \\
& \leq C \mathrm{E}\left(Z_{i}\right) \mathrm{E}\left(N_{j}^{2}\right) \\
& =C \mathrm{E}\left(Z_{i}\right)\left(\mathrm{E}\left(N_{j}\right)+r_{j}^{2}\right) \\
& \leq C \mathrm{E}\left(Z_{i}\right)\left(\mathrm{E}\left(N_{j}\right)+\varepsilon_{n, 1} r_{j}\right),
\end{aligned}
$$


where $N_{j}$ is the Poisson distribution with mean $r_{j}$. In the second case, $\mathrm{E}\left(Z_{j} Z_{i}^{2}\right)$ is bounded (according to (67)) by

$$
\begin{aligned}
\mathrm{E}\left(Z_{j} Z_{i}^{2}\right) & \leq C \mathrm{E}\left(N_{j} Z_{i}^{2}\right) \\
& \leq C \mathrm{E}\left(N_{j}\right) \mathrm{E}\left(Z_{i}^{2}\right) \\
& =C \mathrm{E}\left(N_{j}\right) \sum_{m=1}^{\infty} \mathrm{E}\left(Z_{i}^{2} \mid N_{i}=m\right) \mathrm{e}^{-r_{i}} \frac{r_{i}^{m}}{m !} \\
& \leq C \mathrm{E}\left(N_{j}\right) \sum_{m=1}^{\infty} \mathrm{E}\left(Z_{i} \mid N_{i}=m\right) m \mathrm{e}^{-r_{i}} \frac{r_{i}^{m}}{m !} \\
& \leq C \mathrm{E}\left(N_{j}\right)\left(\mathrm{E}\left(Z_{i} \mid N_{i}=1\right) \mathrm{e}^{-r_{i}} r_{i}+\sum_{m=2}^{\infty} m^{2} \mathrm{e}^{-r_{i}} \frac{r_{i}^{m}}{m !}\right) \\
& \leq C \mathrm{E}\left(N_{j}\right)\left(\mathrm{E}\left(Z_{i}\right)+r_{i}^{2}\right) \\
& \leq C \mathrm{E}\left(N_{j}\right)\left(\mathrm{E}\left(Z_{i}\right)+\varepsilon_{n, 1} r_{i}\right) .
\end{aligned}
$$

We can handle the third case in the same way:

$$
\mathrm{E}\left(Z_{k} Z_{i}^{2}\right) \leq C \mathrm{E}\left(N_{k}\right)\left(\mathrm{E}\left(Z_{i}\right)+r_{i}^{2}\right) \leq C \mathrm{E}\left(N_{k}\right)\left(\mathrm{E}\left(Z_{i}\right)+\varepsilon_{n, 1} r_{i}\right) .
$$

If the three indices are equal then, according to (70), we have

$$
\mathrm{E}\left(Z_{i}^{3}\right) \leq \mathrm{E}\left(Z_{i}\right)+C r_{i}^{2} \leq \mathrm{E}\left(Z_{i}\right)+C \varepsilon_{n, 1} r_{i} .
$$

Now consider the term $\mathrm{E}\left(Z_{i}\right) \mathrm{E}\left(Z_{j} Z_{k}\right)$. If $i, j$, and $k$ are mutually distinct then, according to (67),

$$
\mathrm{E}\left(Z_{i}\right) \mathrm{E}\left(Z_{j} Z_{k}\right) \leq C \mathrm{E}\left(Z_{i}\right) \mathrm{E}\left(N_{j} Z_{k}\right) \leq C \mathrm{E}\left(Z_{i}\right) \mathrm{E}\left(N_{j}\right) \mathrm{E}\left(Z_{k}\right) \leq C \mathrm{E}\left(Z_{i}\right) \mathrm{E}\left(N_{j}\right) \mathrm{E}\left(N_{k}\right) .
$$

If two of the three indices are equal, then there are again three cases to consider: according to (67), we have

$$
\begin{aligned}
\mathrm{E}\left(Z_{i}\right) \mathrm{E}\left(Z_{j}^{2}\right) & \leq C \mathrm{E}\left(Z_{i}\right)\left(\mathrm{E}\left(Z_{j}\right)+C r_{j}^{3}\right) \leq C \mathrm{E}\left(Z_{i}\right)\left(\mathrm{E}\left(N_{j}\right)+C r_{j}^{3}\right), \\
\mathrm{E}\left(Z_{i}\right) \mathrm{E}\left(Z_{j} Z_{i}\right) & \leq C \mathrm{E}\left(Z_{i}\right) \mathrm{E}\left(N_{j} Z_{i}\right) \leq C\left(\mathrm{E}\left(Z_{i}\right)\right)^{2} \mathrm{E}\left(N_{j}\right) \leq C\left(\mathrm{E}\left(Z_{i}\right)+C r_{i}^{2}\right) \mathrm{E}\left(N_{j}\right), \\
\mathrm{E}\left(Z_{i}\right) \mathrm{E}\left(Z_{i} Z_{k}\right) & \leq C\left(\mathrm{E}\left(Z_{i}\right)+C r_{i}^{2}\right) \mathrm{E}\left(N_{k}\right) .
\end{aligned}
$$

If all three indices are equal then, according to (70), we have

$$
\mathrm{E}\left(Z_{i}\right) \mathrm{E}\left(Z_{i}^{2}\right) \leq \mathrm{E}\left(Z_{i}\right)\left(\mathrm{E}\left(Z_{i}\right)+C r_{i}^{3}\right) \leq C \mathrm{E}\left(Z_{i}\right)\left(\mathrm{E}\left(N_{i}\right)+C r_{i}^{3}\right)
$$

Now consider the term $\mathrm{E}\left(Z_{j}\right) \mathrm{E}\left(Z_{i} Z_{k}\right)$. If the indices are mutually distinct then, according to (67), we have

$$
\mathrm{E}\left(Z_{j}\right) \mathrm{E}\left(Z_{i} Z_{k}\right) \leq C \mathrm{E}\left(Z_{j}\right) \mathrm{E}\left(Z_{i} N_{k}\right) \leq C \mathrm{E}\left(Z_{j}\right) \mathrm{E}\left(Z_{i}\right) \mathrm{E}\left(N_{k}\right) \leq C \mathrm{E}\left(N_{j}\right) \mathrm{E}\left(Z_{i}\right) \mathrm{E}\left(N_{k}\right) .
$$


If two of the three indices are equal then there are three cases to consider: according to (67), we have

$$
\begin{aligned}
\mathrm{E}\left(Z_{j}\right) \mathrm{E}\left(Z_{i} Z_{j}\right) & \leq C \mathrm{E}\left(Z_{j}\right) \mathrm{E}\left(Z_{i} N_{j}\right) \leq C \mathrm{E}\left(Z_{j}\right) \mathrm{E}\left(Z_{i}\right) \mathrm{E}\left(N_{j}\right) \leq C \mathrm{E}\left(Z_{i}\right)\left(\mathrm{E}\left(N_{j}\right)\right)^{2}, \\
\mathrm{E}\left(Z_{j}\right) \mathrm{E}\left(Z_{i}^{2}\right) & \leq \mathrm{E}\left(Z_{j}\right)\left(\mathrm{E}\left(Z_{i}\right)+C r_{i}^{3}\right) \leq C \mathrm{E}\left(N_{j}\right)\left(\mathrm{E}\left(Z_{i}\right)+C r_{i}^{3}\right), \\
\mathrm{E}\left(Z_{i}\right) \mathrm{E}\left(Z_{i} Z_{k}\right) & \leq C \mathrm{E}\left(Z_{i}\right) \mathrm{E}\left(Z_{i} N_{k}\right) \leq C\left(\mathrm{E}\left(Z_{i}\right)\right)^{2} \mathrm{E}\left(N_{k}\right) \leq C \mathrm{E}\left(Z_{i}\right) \mathrm{E}\left(N_{i}\right) \mathrm{E}\left(N_{k}\right) .
\end{aligned}
$$

If all three indices are equal then, according to (70), we have

$$
\mathrm{E}\left(Z_{i}\right) \mathrm{E}\left(Z_{i}^{2}\right) \leq \mathrm{E}\left(Z_{i}\right)\left(\mathrm{E}\left(Z_{i}\right)+C r_{i}^{3}\right) \leq C \mathrm{E}\left(Z_{i}\right)\left(\mathrm{E}\left(N_{i}\right)+C r_{i}^{3}\right) .
$$

Now consider the term $\mathrm{E}\left(Z_{k}\right) \mathrm{E}\left(Z_{i} Z_{j}\right)$. If the indices are mutually distinct then, according to (67), we have

$$
\mathrm{E}\left(Z_{k}\right) \mathrm{E}\left(Z_{i} Z_{j}\right) \leq C \mathrm{E}\left(Z_{k}\right) \mathrm{E}\left(Z_{i} N_{j}\right) \leq C \mathrm{E}\left(Z_{k}\right) \mathrm{E}\left(Z_{i}\right) \mathrm{E}\left(N_{j}\right) \leq C \mathrm{E}\left(N_{k}\right) \mathrm{E}\left(Z_{i}\right) \mathrm{E}\left(N_{j}\right) .
$$

If two of the three indices are equal then there are three cases to consider: according to (67), we have

$$
\begin{aligned}
\mathrm{E}\left(Z_{j}\right) \mathrm{E}\left(Z_{i} Z_{j}\right) & \leq C \mathrm{E}\left(Z_{j}\right) \mathrm{E}\left(Z_{i} N_{j}\right) \leq C \mathrm{E}\left(Z_{j}\right) \mathrm{E}\left(Z_{i}\right) \mathrm{E}\left(N_{j}\right) \leq C \mathrm{E}\left(Z_{i}\right)\left(\mathrm{E}\left(N_{j}\right)\right)^{2}, \\
\mathrm{E}\left(Z_{i}\right) \mathrm{E}\left(Z_{i} Z_{j}\right) & \leq C \mathrm{E}\left(Z_{i}\right) \mathrm{E}\left(Z_{i} N_{j}\right) \leq C\left(\mathrm{E}\left(Z_{i}\right)\right)^{2} \mathrm{E}\left(N_{j}\right) \leq C \mathrm{E}\left(Z_{i}\right) \mathrm{E}\left(N_{i}\right) \mathrm{E}\left(N_{j}\right), \\
\mathrm{E}\left(Z_{k}\right) \mathrm{E}\left(Z_{i}^{2}\right) & \leq \mathrm{E}\left(Z_{k}\right)\left(\mathrm{E}\left(Z_{i}\right)+C r_{i}^{3}\right) \leq C \mathrm{E}\left(N_{k}\right)\left(\mathrm{E}\left(Z_{i}\right)+C r_{i}^{3}\right) .
\end{aligned}
$$

If all three indices are equal then, according to (70), we have

$$
\mathrm{E}\left(Z_{i}\right) \mathrm{E}\left(Z_{i}^{2}\right) \leq \mathrm{E}\left(Z_{i}\right)\left(\mathrm{E}\left(Z_{i}\right)+C r_{i}^{3}\right) \leq C \mathrm{E}\left(Z_{i}\right)\left(\mathrm{E}\left(N_{i}\right)+C r_{i}^{3}\right) .
$$

Now consider the term $\mathrm{E}\left(Z_{i}\right) \mathrm{E}\left(Z_{j}\right) \mathrm{E}\left(Z_{k}\right)$. If the indices are mutually distinct then, according to (67), we have

$$
\mathrm{E}\left(Z_{i}\right) \mathrm{E}\left(Z_{j}\right) \mathrm{E}\left(Z_{k}\right) \leq C \mathrm{E}\left(Z_{i}\right) \mathrm{E}\left(N_{j}\right) \mathrm{E}\left(N_{k}\right)
$$

If two of the indices are equal then there are three cases to consider: according to (67), we have

$$
\begin{aligned}
& \mathrm{E}\left(Z_{i}\right) \mathrm{E}\left(Z_{j}\right) \mathrm{E}\left(Z_{j}\right) \leq C \mathrm{E}\left(Z_{i}\right) \mathrm{E}\left(N_{j}\right) \mathrm{E}\left(N_{j}\right), \\
& \mathrm{E}\left(Z_{i}\right) \mathrm{E}\left(Z_{j}\right) \mathrm{E}\left(Z_{i}\right) \leq C \mathrm{E}\left(Z_{i}\right) \mathrm{E}\left(N_{j}\right) \mathrm{E}\left(N_{i}\right), \\
& \mathrm{E}\left(Z_{i}\right) \mathrm{E}\left(Z_{i}\right) \mathrm{E}\left(Z_{k}\right) \leq C \mathrm{E}\left(Z_{i}\right) \mathrm{E}\left(N_{i}\right) \mathrm{E}\left(N_{k}\right) .
\end{aligned}
$$

If all three indices are equal then, according to (70), we have

$$
\mathrm{E}\left(Z_{i}\right) \mathrm{E}\left(Z_{i}\right) \mathrm{E}\left(Z_{i}\right) \leq C \mathrm{E}\left(Z_{i}\right) \mathrm{E}\left(N_{i}\right) \mathrm{E}\left(N_{i}\right) .
$$

Thus, by (66)-(91) we have

$$
\begin{aligned}
& \left|\mathrm{E}\left(h\left(\frac{L_{n}^{\prime \prime}-\mathrm{E}\left(L_{n}^{\prime \prime}\right)}{\left(\operatorname{var}\left(L_{n}^{\prime \prime}\right)\right)^{1 / 2}}\right)\right)-\mathrm{E}(h(N))\right| \\
& \quad \leq C \frac{\mathrm{E}\left(L_{n}^{\prime \prime}\right)\left(Q_{n}^{2}+Q_{n}+1+\varepsilon_{n, 1}^{2}+\varepsilon_{n, 1}+Q_{n} \varepsilon_{n, 1}+\varepsilon_{n, 2}\right)+Q_{n} \varepsilon_{n, 2}+\varepsilon_{n, 2}}{\left(\operatorname{var}\left(L_{n}^{\prime \prime}\right)\right)^{3 / 2}} .
\end{aligned}
$$




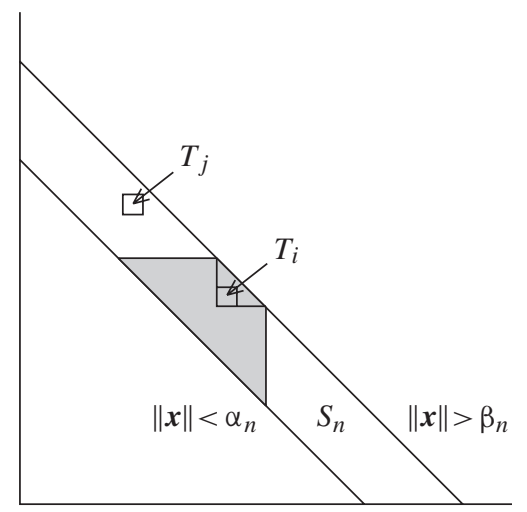

FiguRe 1: For $S_{n}:=\left\{\boldsymbol{x}: \alpha_{n}<\|\boldsymbol{x}\|<\beta_{n}\right\}$, and for $T_{i}$ and $T_{j}$ with $T_{i} \cap S_{n} \neq \varnothing$ and $T_{j} \cap S_{n} \neq \varnothing$, $Z_{i}$ and $Z_{j}$ are independent if $T_{j}$ and the dark region generated by $T_{i}$ have no overlapping region.

By splitting $\mathbb{R}_{+}^{d}$ into very small cubes, $T_{i}$, i.e. by choosing $l$ to be very small, we can make $\varepsilon_{n, 1}$ and $\varepsilon_{n, 2}$ arbitrarily small. Therefore, we have

$$
\left|\mathrm{E}\left(h\left(\frac{L_{n}^{\prime \prime}-\mathrm{E}\left(L_{n}^{\prime \prime}\right)}{\left(\operatorname{var}\left(L_{n}^{\prime \prime}\right)\right)^{1 / 2}}\right)\right)-\mathrm{E}(h(N))\right| \leq C \frac{\mathrm{E}\left(L_{n}^{\prime \prime}\right)\left(Q_{n}^{2}+Q_{n}+1\right)+1}{\left(\operatorname{var}\left(L_{n}^{\prime \prime}\right)\right)^{3 / 2}} .
$$

Now we need only to estimate the quantities $\mathrm{E}\left(L_{n}^{\prime \prime}\right), Q_{n}$, and $\operatorname{var}\left(L_{n}^{\prime \prime}\right)$. First we estimate $\mathrm{E}\left(L_{n}^{\prime \prime}\right)$ : by $(61)$ we have

$$
\begin{aligned}
\mathrm{E}\left(L_{n}^{\prime \prime}\right) & \leq \mathrm{E}\left(L_{n}^{\prime}\right)+C n^{-1}(\log n)^{2(d-1)}(\log \log n)^{2} \\
& \leq \mathrm{E}\left(L_{n}\right)+C(\log n)^{(d-2)-(b-1)(d-1)}+C(\log n)^{-(a-(d-1))} \quad(\text { by }(55)) \\
& \leq C(\log n)^{d-2} \quad(\text { by Theorem } 1) .
\end{aligned}
$$

Now consider $Q_{n}$. As we see in Figure $1, Z_{j}$ is independent of $Z_{i}$ if $T_{j}$ and the dark region generated by $T_{i}$ have no overlapping region. Thus,

$$
Q_{n} \leq C n \mathrm{e}^{-\alpha_{n}}\left(\beta_{n}-\alpha_{n}\right)^{d} \leq C(\log \log n)^{d+1} .
$$

Finally, with the choice of $b$ in (40), we have the following estimate for $\operatorname{var}\left(L_{n}^{\prime \prime}\right)$ :

$$
\begin{aligned}
\operatorname{var}\left(L_{n}^{\prime \prime}\right) & =\mathrm{E}\left(L_{n}^{\prime \prime}\left(L_{n}^{\prime \prime}-1\right)\right)+\mathrm{E}\left(L_{n}^{\prime \prime}\right)-\left(\mathrm{E}\left(L_{n}^{\prime \prime}\right)\right)^{2} \\
& =\mathrm{E}\left(L_{n}^{\prime}\left(L_{n}^{\prime}-1\right)\right)+\mathrm{E}\left(L_{n}^{\prime}\right)-\left(\mathrm{E}\left(L_{n}^{\prime}\right)\right)^{2}+O\left(n^{-1}(\log n)^{3(d-1)}(\log \log n)^{3}\right) \\
& =\operatorname{var}\left(L_{n}^{\prime}\right)+O\left(n^{-1}(\log n)^{3(d-1)}(\log \log n)^{3}\right),(61),(55), \text { and (2)) } \\
& =\operatorname{var}\left(L_{n}\right)+O\left((\log n)^{2(d-2)-(1 / 2)(b-1)(d-1)}\right)+o\left((\log n)^{d-2}\right) \quad \text { (by (58) and (3)) } \\
& =\operatorname{var}\left(L_{n}\right)+o\left((\log n)^{d-2}\right) \quad(\text { by (40)). }
\end{aligned}
$$

Note that from (95) we also have

$$
\left|\operatorname{var}\left(L_{n}^{\prime \prime}\right)-\operatorname{var}\left(L_{n}^{\prime}\right)\right| \leq C n^{-1}(\log n)^{3(d-1)}(\log \log n)^{3} .
$$


Therefore, by (92), (93), (94), (95), and (3), we have

$$
\left|\mathrm{E}\left(h\left(\frac{L_{n}^{\prime \prime}-\mathrm{E}\left(L_{n}^{\prime \prime}\right)}{\left(\operatorname{var}\left(L_{n}^{\prime \prime}\right)\right)^{1 / 2}}\right)\right)-\mathrm{E}(h(N))\right|=O\left((\log n)^{-(d-2) / 2}(\log \log n)^{d+1}\right) .
$$

This completes the proof of Proposition 1.

To prove Theorem 3, we need the following obvious lemma. We omit its proof.

Lemma 2. Let $r_{n}, r_{n} \rightarrow 0$, be given. If

(i) $d_{\mathrm{TV}}\left(X_{n}, Y_{n}\right)=O\left(r_{n}\right)$,

(ii) $\left|\mathrm{E}\left(X_{n}\right)-\mathrm{E}\left(Y_{n}\right)\right|=O\left(r_{n}\left(\operatorname{var}\left(X_{n}\right)\right)^{1 / 2}\right)$, and

(iii) $\left|\operatorname{var}\left(X_{n}\right)-\operatorname{var}\left(Y_{n}\right)\right|=O\left(r_{n}\left(\operatorname{var}\left(X_{n}\right)\right)^{1 / 2}\right)$,

then

$$
X_{n} \in \mathrm{CLT}\left(r_{n}\right) \text { if and only if } Y_{n} \in \operatorname{CLT}\left(r_{n}\right) .
$$

Proposition 2. For any $r_{n}$ with $r_{n} \rightarrow 0$ but with $r_{n} \geq C(\log n)^{-(d-2) / 2}$, we have

$$
L_{n} \in \operatorname{CLT}\left(r_{n}\right) \text { if and only if } L_{n}^{\prime \prime} \in \operatorname{CLT}\left(r_{n}\right) .
$$

Proof. With the choice of $a$ and $b$ in (40), for any $r_{n}$ with $r_{n} \rightarrow 0$ but with

$$
r_{n} \geq C(\log n)^{-(d-2) / 2},
$$

the proposition follows from Lemma 2, (60), (61), (96), (48), (55), and (58).

Our final lemma relates the estimates of the rate of weak convergence using the $d_{1}$ metric (defined in (65)) and weak convergence in the sense of CLT $\left(r_{n}\right)$ (defined in (6)).

Lemma 3. Let $\left(\xi_{n}, n \geq 1\right)$ be a sequence of random variables with finite second moments, and let $\bar{\xi}_{n}:=\left(\xi_{n}-\mathrm{E}\left(\xi_{n}\right)\right) / \sqrt{\operatorname{var}\left(\xi_{n}\right)}$. If $d_{1}\left(\bar{\xi}_{n}, N\right)=O\left(r_{n}\right)$, where $N$ has a standard normal distribution and where $r_{n}>0$ with $r_{n} \rightarrow 0$ as $n \rightarrow \infty$, then $\xi_{n} \in \operatorname{CLT}\left(\sqrt{r_{n}}\right)$.

Proof. Set $a_{n}=\sqrt{r_{n}}$. Given an $x \in \mathbb{R}$ and an $n$, set $y=x+a_{n}$. Define the bounded, continuous, piecewise-linear function $h$ on $\mathbb{R}$ by

$$
h(t)= \begin{cases}a_{n}, & t \leq x \\ y-t, & x \leq t \leq y, \\ 0, & t \geq y\end{cases}
$$

Then, for $n$ sufficiently large that $a_{n}<1$, we have $|h(t)| \leq 1$ for all $t$ and $\left|h^{\prime}(t)\right| \leq 1$ for all $t$ except $t=x$ and $t=y$. Thus, $h$ can be approximated uniformly by continuously differentiable functions $g$ with $|g(t)| \leq 1$ and $\left|g^{\prime}(t)\right| \leq 1$ for all $t$. Hence,

$$
|\mathrm{E}(h(X))-\mathrm{E}(h(Y))| \leq 2\left|\mathrm{E}\left(\frac{1}{2} h(X)\right)-\mathrm{E}\left(\frac{1}{2} h(Y)\right)\right| \leq 2 d_{1}(X, Y)
$$

for any pair of random variables $X$ and $Y$. By the choice of $h$, for all $X$ we have

$$
a_{n} \mathrm{P}(X \leq x) \leq \mathrm{E}(h(X)) \leq a_{n} \mathrm{P}(X \leq y) .
$$


Hence, if $d_{1}\left(\bar{\xi}_{n}, N\right)=O\left(r_{n}\right)$, there is a constant $C$ such that

$$
\begin{aligned}
a_{n} \mathrm{P}\left(\bar{\xi}_{n} \leq x\right) & \leq \mathrm{E}\left(h\left(\bar{\xi}_{n}\right)\right) \\
& \leq \mathrm{E}(h(N))+C r_{n} \\
& \leq a_{n} \mathrm{P}\left(N \leq x+a_{n}\right)+C r_{n} \\
& \leq a_{n} \mathrm{P}(N \leq x)+C a_{n}^{2}+C r_{n} .
\end{aligned}
$$

By the choice of $a_{n}$, we then have

$$
\mathrm{P}\left(\bar{\xi}_{n} \leq x\right) \leq \mathrm{P}(N \leq x)+2 C \sqrt{r_{n}} .
$$

Here the choice of $C$ can be made independently of $n$ and $x$. For inequality in the other direction, note that there is a constant $C$ (independent of $n$ and $y$ ) such that

$$
\begin{aligned}
a_{n} \mathrm{P}(N \leq y) & \leq a_{n} \mathrm{P}(N \leq x)+C a_{n}^{2} \\
& \leq \mathrm{E}(h(N))+C a_{n}^{2} \\
& \leq \mathrm{E}\left(h\left(\bar{\xi}_{n}\right)\right)+C r_{n}+C a_{n}^{2} \\
& \leq a_{n} \mathrm{P}\left(\bar{\xi}_{n} \leq y\right)+C\left(r_{n}+a_{n}^{2}\right) .
\end{aligned}
$$

Again by the choice of $a_{n}$ we have

$$
\mathrm{P}(N \leq y) \leq \mathrm{P}\left(\bar{\xi}_{n} \leq y\right)+2 C \sqrt{r_{n}} .
$$

Combining (97) with (98) yields $\xi \in \operatorname{CLT}\left(\sqrt{r_{n}}\right)$.

Theorem 3 now follows from Propositions 1 and 2 and Lemma 3.

\section{Acknowledgements}

This work was partially done while all three authors were visiting the Institute for Mathematical Sciences, National University of Singapore, in 2003 and finalized while the second author was visiting the Department of Mathematics, Northeast Normal University. We thank Louis Chen for providing the first opportunity.

The work of the first author was supported by NSFC Grant 10571020 and NUS Grant R-155-000-056-112, that of the second author was supported by Korea Research Foundation Grant KRF-2005-015-C00049, and that of the third author was supported by the Isaac Newton Institute for Mathematical Sciences, Cambridge.

\section{References}

[1] Bai, Z.-D., ChaO, C.-C., Hwang, H.-K. And Liang, W.-Q. (1998). On the variance of the number of maxima in random vectors and its applications. Ann. Appl. Prob. 8, 886-895.

[2] Bai, Z.-D., Devroye, L., Hwang, H.-K. and Tsai, T.-H. (2005). Maxima in hypercubes. Random Structures Algorithms 27, 290-309.

[3] BAI, Z.-D. et al. (2001). Limit theorems for the number of maxima in random samples from planar regions. Electron. J. Prob. 6, 41pp.

[4] Barbour, A. D. and Xia, A. (2001). The number of two-dimensional maxima. Adv. Appl. Prob. 33, 727-750.

[5] Baryshnikov, Y. (2000). Supporting points processes and some of their applications. Prob. Theory Relat. Fields 117, 163-182.

[6] Bhatt, A. And Roy, R. (2004). On a random directed spanning tree. Adv. Appl. Prob. 36, 19-42. 
[7] BräKER, H. AND Hsing, T. (1998). On the area and perimeter of a random convex hull in a bounded convex set. Prob. Theory Relat. Fields 111, 517-550.

[8] Groeneboom, P. (1988). Limit theorems for convex hulls. Prob. Theory Relat. Fields 79, 329-368.

[9] Hall, P. (1992). The Bootstrap and Edgeworth Expansion. Springer, New York.

[10] Hsing, T. (1994). On the asymptotic distribution of the area outside a random convex hull in a disk. Ann. Appl. Prob. 4, 478-493.

[11] Janson, S., ŁuczaK, T. And Ruciński, A. (2000). Random Graphs. John Wiley, New York.

[12] Kesten, H. AND LeE, S. (1996). The central limit theorem for weighted minimal spanning trees on random points. Ann. Appl. Prob. 6, 495-527.

[13] Penrose, M. D. (2003). Random Geometric Graphs (Oxford Studies Prob. 6). Oxford University Press.

[14] Penrose, M. D. and Wade, A. (2004). Random minimal directed spanning trees and Dickman-type distributions. Adv. Appl. Prob. 36, 691-714.

[15] Penrose, M. D. And Yukich, J. E. (2001). Central limit theorems for some graphs in computational geometry. Ann. Appl. Prob. 11, 1005-1041.

[16] Penrose, M. D. And Yukich, J. E. (2003). Weak laws of large numbers in geometric probability. Ann. Appl. Prob. 13, 277-303.

[17] Steele, J. M. (1997). Probability Theory and Combinatorial Optimization. Society for Industrial and Applied Mathematics, Philadelphia, PA.

[18] Yukich, J. E. (1998). Probability Theory of Classical Euclidean Optimization Problems (Lecture Notes Math. 1675). Springer, Berlin. 